\title{
ON THE REDUCTION OF THE GENERAL ABELIAN INTEGRAL*
}

BY

\author{
J. C. FIELDS
}

\section{$\S 1$.}

In their exceedingly interesting book Théorie des Fonctions algébriques et de leurs Intégrales, pp. 344-345, APPell and Goursat have given a brief sketch of Hermite's method for obtaining by rational operations the reduced form for a hyperelliptic integral, prefacing the same by a remark which would seem to imply that the more general problem in the case of the Abelian integrals was still awaiting its solution.

To this remark was due an attempt on my part to solve the indicated problem, and the method by which I succeeded in effecting its solution will be found in the present paper. The results here given, it may be remarked, have been in my possession now for about four years and were presented at the annual meeting of the American Mathematical Society held in Toronto in 1897 but have not hitherto been published.

Let

$$
F(z, u)=\sum e_{r s} z^{r} u^{s}=0
$$

be the equation to an irreducible algebraic curve of degree $n$.

The form of this equation we shall suppose to be the standard one in which the only multiple points are double points separate from the branch points and with distinct tangents. We shall throughout regard $u$ as the dependent variable and shall further assume that no line parallel to the axis of $u$ is an inflexional tangent or includes more than one point of the nature of a branch or double point or is tangent at a double point, the singular points too being all supposed to be finite and the asymptotes all distinct and none of them parallel to an axis and no two parallel to each other.

That the most general algebraic equation can be reduced to such a form by a birational transformation is a well-known fact, and in such a reduced form it is customary to treat the equation.

* Presented to the Society at the Toronto meeting, August 16-17, 1897, under a different title. Receired for publication November 28, 1900. 
On the above hypotheses a term in $u^{n}$ will present itself in the equation to our curve and we shall find it convenient to assume that its coefficient $e_{0 n}$ has the value 1.

Also as a consequence of the said hypotheses $z$ and $u$ can only become infinite together, and further for any given value $z=a$ the equation $F(a, u)=0$ will never furnish less than $n-1$ distinct values of $u$.

On indicating any arbitrary rational function of $z, u$ by $R(z, u)$ the general Abelian integral corresponding to our curve $F(z, u)=0$ will be

$$
\int R(z, u) d z \text {. }
$$

This integral we know may be expressed as the sum of a rational function of $z, u$, of an integral of the first kind, of a linear function with constant coeffi. cients of $p$ elementary integrals of the second kind, and of a number of elementary integrals of the third kind, where

$$
p=\frac{1}{2}(n-1)(n-2)-d
$$

is the number known as the "deficiency" of the curve, $d$ indicating the number of the double points.

Starting out now with any arbitrary Abelian integral we purpose in this paper to reduce the same to a sum of the character in question, employing thereto a succession of processes, none of which involves other than rational operations in our variables.

By aid of the relation $F(z, u)=0$ it is known that any rational function $R(z, u)$ may be reduced to the form of a polynomial in $u$ of the degree $n-1$, whose coefficients are rational functions of $z$, and on applying the principle of partial fractions to these functions of $z$ we may express $R(z, u)$ in the form :

$$
R(z, u)=\sum_{\lambda} R_{\lambda}\left(\frac{1}{z-a_{\lambda}}, u\right)+R_{0}(z, u),
$$

where $R_{\lambda}\left(1 /\left(z-a_{\lambda}\right), u\right)$ denotes a polynomial in $1 /\left(z-a_{\lambda}\right), u$ and $R_{0}(z, u)$ a polynomial in $z, u$, the degrees of these polynomials in the variable $u$ not being greater than $n-1$.

Our Abelian integral then takes the form:

$$
\int R(z, u) d z=\sum_{\lambda} \int R_{\lambda}\left(\frac{1}{z-a_{\lambda}}, u\right) d z+\int R_{0}(z, u) d z .
$$

We shall assume that $R_{\lambda}\left(1 /\left(z-a_{\lambda}\right), u\right)$ contains only negative powers of $z-a_{\lambda}$, as all terms.involving other than such powers may be supposed to be included under $R_{0}(z, u)$. 


\section{$\S 2$.}

For the further reduction of our integral we have need of a formula which we shall now derive. The formula in question is the following:

$$
\frac{d u}{d z}=-\frac{F_{z}^{\prime}}{F_{u}^{\prime}}=\sum_{e} \frac{\phi_{e}(u)}{z-a_{e}}=\sum_{e} \frac{c_{e} F\left(a_{e}, u\right)}{\left(z-a_{e}\right)\left(u-b_{e}\right)},
$$

where the summation is supposed to extend to all branch and double points $\left(a_{e}, b_{e}\right)$, and where for the constant coefficients $c$ we have

$$
c_{e}=\left[\frac{1}{F_{u}^{\prime \prime}\left(a_{e}, u\right)}\right]_{u=b_{e}} \text { or }\left[\frac{2}{F_{u}^{\prime \prime}\left(a_{e}, u\right)}\right]_{u=b_{e}}
$$

according as $\left(a_{e}, b_{e}\right)$ is a branch or double point.

Starting out from the identity

$$
-\frac{F_{z}^{\prime}}{F_{u}^{\prime \prime}}=-\frac{F_{z}^{\prime}\left[F_{u}^{\prime}\right]_{u=u_{1}} \cdots\left[F_{u}^{\prime}\right]_{u=u_{n-1}}}{F_{u}^{\prime}\left[F_{u}^{\prime}\right]_{u=u_{1}} \cdots\left[F_{u}^{\prime}\right]_{u=u_{n-1}}},
$$

where $u_{1}, u_{2}, \cdots, u_{n-1}$ are the $n-1$ roots of $F(z, u)=0$ conjugate to $u$, we see that the denominator of the expression on the right is a symmetric function of the $n$ quantities $u, u_{1}, \cdots, u_{n-1}$ and therefore a rational function of $z$. It is, in fact, the discriminant, an integral rational function of $z$ of degree $n(n-1)$ whose factórs $z-a_{e}$ correspond to the several branch and double points. In case a double point corresponds to the value $z=a_{e}$ the factor $z-a_{e}$ will appear to the second power in the discriminant and in the case of a branch point to the first power, as is well known.

The factor $\left[F_{u}^{\prime}\right]_{u=u_{1}} \cdots\left[F_{u}^{\prime}\right]_{u=u_{n-1}}$ in the numerator of the above expression is symmetric in the $n-1$ quantities $u_{1} \cdots u_{n-1}$ and may evidently be expressed as a polynomial in $z, u$. The numerator itself may then be written in the form :

$$
A_{1} u^{n-1}+A_{2} u^{n-2}+\cdots+A_{n},
$$

where the coefficients $A$ are polynomials in $z$.

On indicating the discriminant by the letter $D$ we then obtain for $d u^{\prime} d z$ the expression

$$
\frac{d u}{d z}=\frac{A_{1} u^{n-1}+A_{2} u^{n-2}+\cdots+A_{n}}{D},
$$

where, as may readily be shown, the degree of the numerator in $z, u$ is not greater than $n(n-1)$ and where in particular the degree of the term $A_{n}$ is less than this number.

Suppose, namely, that the directions of the $n$ points at $\infty$ on the curve $F(z, u)=0$ are given by the equations 


$$
u-\kappa_{1} z=0, u-\kappa_{2} z=0, \ldots, u-\kappa_{n} z=0,
$$

where, according to our original hypothesis in regard to the asymptotes of our curve, the $n$ coefficients $\kappa$ all have different values. The differential coefficient $d u / d z$ will then have the values $\kappa_{1}, \kappa_{2}, \ldots, \kappa_{n}$, respectively, for the several branches at $\infty$.

Indicating the degree of the numerator in the expression on the right of (6) by $\nu$ and representing by $a_{1}, a_{2}, \cdots, a_{n}$ the coefficients of the terms of this degree in the elements $A_{1} u^{n-1}, A_{2} u^{n-2}, \ldots, A_{n}$ respectively, we shall have

$$
a_{1} \kappa_{r}^{n-1}+a_{2} \kappa_{r}^{n-2}+\cdots+a_{n}
$$

for the coefficient of $z^{\nu}$ in the numerator in question, on substituting for $u$ in terms of $z$ from the $r$ th branch of the curve. Now in case we had $\nu>n(n-1)$ the differential coefficient in (6) would be infinite for some branch of the curve at $\infty$ unless the expression (8) be equal to 0 for each of the $n$ values $\kappa_{1}, \kappa_{2}$, $\cdots, \kappa_{n}$ of $\kappa_{r}$. In this case, however, the expression in question being only of the degree $n-1$ must vanish identically and we should have $a_{1}=a_{2}=\cdots=a_{n}=0$. It follows therefore that $\nu$ cannot be greater than $n(n-1)$.

Assuming then that we have $\nu=n(n-1)$, substituting for $u$ in terms of $z$ from the equation to one of the $n$ branches at $\infty$, and putting $z=\infty$ in (6), we derive

$$
\kappa_{r}=\frac{a_{1}}{\delta} \kappa_{r}^{n-1}+\frac{a_{2}}{\delta} \kappa_{r}^{n-2}+\cdots+\frac{a_{n}}{\delta},
$$

where $\delta$ is the coefficient of $z^{n(n-1)}$ in $D$.

Now this equation is of the degree $n-1$ in $\kappa_{r}$ and must be satisfied by the $n$ quantities $\kappa_{1}, \kappa_{2}, \cdots, \kappa_{n}$. It must therefore be an identity, and we consequently have

$$
a_{1}=0, a_{2}=0, \cdots, a_{n-2}=0, a_{n-1}=\delta, a_{n}=0 .
$$

We see therefore that the degree of the coefficient $A_{n}$, as well as that of the other coefficients $\dot{A}$ in the numerator of the expression to the right of (6), must be less than $n(n-1)$. On applying to this expression the principle of partial fractions, the various parts will evidently all be fractional in their character.

The expression in (6) may then be written in the form :

$$
\frac{d u}{d z}=\sum_{e}\left\{\frac{\phi_{e}(u)}{z-a_{e}}+\frac{\psi_{e}(u)}{\left(z-a_{e}\right)^{2}}\right\},
$$

where the summation extends to all those values $z=a_{e}$ to which correspond branch or double points, and where the numerators $\phi(u)$ and $\psi(u)$ are polynomials in $u$ of degree $n-1$.

We have already remarked that $z-a_{e}$ is a quadratic factor of the discriminant $D$ in case a double point corresponds to the value $z=a_{e}$, whereas in the case of a branch point the corresponding factor enters only to the first power. 
In the summation above, then, the numerator $\psi_{e}(u)$ in an element corresponding to a branch point must evidently be 0 , and we shall prove that this also is so in the case of an element corresponding to a double point.

Consider, namely, an element

$$
\frac{\phi(u)}{z-a}+\frac{\psi(u)}{(z-a)^{2}}
$$

where to $z=a$ corresponds a double point. The other points of our curve corresponding to this value of the variable will then be ordinary points by virtue of our original hypothesis, and the differential coefficient $d u / d z$ will evidently be finite for each of the $n-1$ points corresponding to the value $z=a$. Its values for the two branches at the double point I shall indicate by $m_{1}$ and $m_{2}$ respectively.

For the $n-1$ points corresponding to the value $z=a$ the summation in (10) and therewith the element here in question must be finite. It evidently follows that $\psi(u)$ must be equal to 0 for each of the $n-1$ values of $u$ corresponding to $z=a$, and on writing

$$
F(a, u)=(u-b)^{2}\left(u-b^{\prime}\right)\left(u-b^{\prime \prime}\right) \cdots\left(u-b^{(n-2)}\right),
$$

we must have

$$
\psi(u)=c(u-b)\left(u-b^{\prime}\right) \cdots\left(u-b^{(n-2)}\right),
$$

where $c$ is a constant, for $\psi(u)$ has the degree $n-1$.

We may therefore write

$$
\psi(u)=\frac{c \mathscr{F}(a, u)}{u-b},
$$

and our element will have the form

$$
\frac{\phi(u)}{z-a}+\frac{\psi(u)}{(z-a)^{2}}=\frac{1}{z-a}\left\{\phi(u)+\frac{c F(a, u)}{(z-a)(u-b)}\right\} .
$$

Now in order that this element may be finite for the two branches at the double point it is necessary that the expression in parentheses should vanish for both these branches.

Writing this expression in the form

$$
\phi(u)+\frac{u-b}{z-a} \frac{c F(a, u)}{(u-b)^{2}}
$$

we see then that we must have

$$
\begin{aligned}
& \phi(b)+m_{1}\left[\frac{c F(a, u)}{(u-b)^{2}}\right]_{u=b}=0, \\
& \phi(b)+m_{2}\left[\frac{c F(a, u)}{(u-b)^{2}}\right]_{u=b}=0 .
\end{aligned}
$$


From this it follows that

and as we have

$$
c\left(m_{1}-m_{2}\right)\left[\frac{F(a, u)}{(u-b)^{2}}\right]_{u=b}=0
$$

we must have $c=0$.

$$
m_{1} \neq m_{2}, \quad\left[\frac{F(a, u)}{(u-b)^{2}}\right]_{u=b} \neq 0
$$

It follows therefore that $\psi(u)=0$ and the summation in (10) reduces to the form

$$
\frac{d u}{d z}=\sum_{e} \frac{\phi_{e}(u)}{z-a_{e}},
$$

where, as before, the summation is supposed to extend to every value $z=a_{e}$ to which corresponds a branch or double point. Now the differential coefficient $d u / d z$ can only become infinite for the branch points, and for a branch point too it can only become infinite like $(z-a)^{-\frac{1}{2}}$, so that we see that in any case the numerator $\phi_{e}(u)$ of an element in the above summation must equal 0 for each of the $n-1$ values of $u$ corresponding to the value $z=a_{e}$. It follows therefrom that $\phi_{e}(u)$ must have the form

$$
\phi_{e}(u)=\frac{c_{e} F\left(a_{e}, u\right)}{u-b_{e}},
$$

and for the differential coefficient $d u / d z$ we obtąin the expression

$$
\frac{d u}{d z}=\sum_{e} \frac{c_{e} F\left(a_{e}, u\right)}{\left(z-a_{e}\right)\left(u-b_{e}\right)},
$$

where it only remains now to determine the value of the constants $c_{e}$.

First considering the case of a double point $\left(a_{e}, b_{e}\right)$ we may evidently write the expression for our differential coefficient in the form

$$
\frac{d u}{d z}=\frac{c_{e} F\left(a_{e}, u\right)}{\left(u-b_{e}\right)^{2}} \cdot \frac{u-b_{e}}{z-a_{e}}+f(z, u),
$$

where the value of the function $f(z, u)$ for the point $\left(a_{e}, b_{e}\right)$ is unambiguous.

On considering the respective values of this expression for the two branches passing through the double point, we shall have

$$
\begin{aligned}
& m_{1}=\left[\frac{c_{e} F\left(a_{e}, u\right)}{\left(u-b_{e}\right)^{2}}\right]_{u=b_{e}} m_{1}+f\left(a_{e}, b_{e}\right)=\frac{1}{2} c_{e} m_{1}\left[F_{u}^{\prime \prime}\right]_{a_{e} b_{e}}+f\left(a_{e}, b_{e}\right), \\
& m_{2}=\left[\frac{c_{e} F\left(a_{e}, u\right)}{\left(u-b_{e}\right)^{2}}\right]_{u=b_{e}} m_{2}+f\left(a_{e}, b_{e}\right)=\frac{1}{2} c_{e} m_{2}\left[F_{u}^{\prime \prime \prime}\right]_{a_{e} b_{e}}+f\left(a_{e}, b_{e}\right) ;
\end{aligned}
$$

whence by subtraction

$$
\left(m_{1}-m_{2}\right)=\frac{1}{2}\left(m_{1}-m_{2}\right) c_{e}\left[F_{u}^{\prime \prime}\right]_{a_{e} b_{e}},
$$


and therefore

$$
c_{e}=\frac{2}{\left[F_{u}^{\prime \prime}\right]_{a_{e} b_{e}}}
$$

In the case of a branch point $\left(a_{e}, b_{e}\right)$ we evidently have

$$
\left[\frac{\left(u-b_{e}\right)^{2}}{z-a_{e}}\right]_{a_{e} b_{e}}=\left[\frac{d}{d z}\left(u-b_{e}\right)^{2}\right]_{a_{e} b_{e}}=\left[2\left(u-b_{e}\right) \frac{d u}{d z}\right]_{a_{e} b_{e}} .
$$

We have however also :

$$
\left[\left(u-b_{e}\right) \frac{d u}{d z}\right]_{a_{e} b_{e}}=\left[\frac{c_{e} F\left(a_{e}, u\right)}{z-a_{e}}\right]_{a_{e} b_{e}}=\left[\frac{c_{e} F\left(a_{e}, u\right)}{\left(u-b_{e}\right)^{2}} \frac{\left(u-b_{e}\right)^{2}}{z-a_{e}}\right]_{a_{e} b_{e}} ;
$$

and from these two formulæ combined we derive :

$$
\left[\frac{\left(u-b_{e}\right)^{2}}{z-a_{e}}\right]_{a_{e} b_{e}}=\left[\frac{2 c_{e} F\left(a_{e}, u\right)}{\left(u-b_{e}\right)^{2}} \frac{\left(u-b_{e}\right)^{2}}{z-a_{e}}\right]_{a_{e} b_{e}} .
$$

Here the expression to the left is evidently finite, and it is also different from 0 since by our original hypothesis the branch point $\left(a_{e}, b_{e}\right)$ is not at the same time a double point. We may therefore divide both sides of our equation by the expression in question, and obtain

$$
1=c_{e}\left[\frac{2 F\left(a_{e}, u_{4}\right)}{\left(u-b_{e}\right)^{2}}\right]_{a_{e} b_{e}}=c_{e}\left[F_{u}^{\prime \prime \prime}\right]_{a_{e} b_{e}}
$$

whence in the case of a branch point $\left(a_{e}, b_{e}\right)$ we have

$$
c_{e}=\frac{1}{\left[F_{u}^{\prime \prime \prime}\right]_{a_{e} b_{e}}} \text {. }
$$

We have therefore established our formula (5).

\section{$\S 3$.}

Returning now to the reduction of our integral in (4) we shall first consider an individual element,

$$
\int R_{\lambda}\left(\frac{1}{z-a_{\lambda}}, u\right) d z
$$

in the summation appearing on the right.

Supposing the highest exponent of $1 /\left(z-a_{\lambda}\right)$ in the expression under the integral sign to be greater than 1 and indicating the same by $r$ we may write :

$$
R_{\lambda}\left(\frac{1}{z-a_{\lambda}}, u\right)=\frac{f_{r}(u)}{\left(z-a_{\lambda}\right)^{r}}+R^{\prime}\left(\frac{1}{z-a_{\lambda}}, u\right),
$$

where $f(u)$ is a polynomial in $u$ of degree $n-1$ and where $R^{\prime}\left(1 /\left(z-a_{\lambda}\right), u\right)$ is a polynomial in $1 /\left(z-a_{\lambda}\right), u$ of degrees $r-1$ and $n-1$ respectively in $1 /\left(z-a_{\lambda}\right)$ and $u$. 
We shall then have:

$$
\begin{aligned}
& \int R_{\lambda}\left(\frac{1}{z-a_{\lambda}}, u\right) d z= \int \frac{f_{r}(u)}{\left(z-a_{\lambda}\right)^{r}} d z+\int R^{\prime}\left(\frac{1}{z-a_{\lambda}}, u\right) d z \\
&= \frac{g_{r-1}(u)}{\left(z-a_{\lambda}\right)^{r-1}}+\int\left\{\frac{f_{r}(u)}{\left(z-a_{\lambda}\right)^{r}}-\frac{d}{d z} \frac{g_{r-1}(u)}{\left(z-a_{\lambda}\right)^{r-1}}\right\} d z \\
& \text { (11) } \quad+\int R^{\prime}\left(\frac{1}{z-a_{\lambda}}, u\right) d z \\
&=\frac{g_{r-1}(u)}{\left(z-a_{\lambda}\right)^{r-1}}+\int\left\{\frac{f_{r}(u)+(r-1) g_{r-1}(u)}{\left(z-a_{\lambda}\right)^{r}}\right. \\
&\left.-\sum_{e} \frac{g_{r-1}^{\prime}(u) \cdot \phi_{e}(u)}{\left(z-a_{\lambda}\right)^{r-1}\left(z-a_{e}\right)}\right\} d z+\int R^{\prime}\left(\frac{1}{z-a_{\lambda}}, u\right) d z ;
\end{aligned}
$$

where $g_{r-1}(u)$ is a polynomial in $u$ of degree $n-1$, as yet undetermined, and where for $d u / d z$ we have substituted its expression as given in (5), so that the summation with regard to $e$ which here appears is supposed to extend to all branch and double points. We shall first suppose that none of the points of the curve corresponding to the value $z=a_{\lambda}$ is a branch or double point. In this case then we shall have $a_{\lambda} \neq a_{e}$ for all values of the $a_{e}$ 's. For the polynomial $g_{r-1}(u)$ then choosing

$$
g_{r-1}(u)=-\frac{f_{r}(u)}{r-1}
$$

we reduce our integral (11) to the form :

$$
\begin{aligned}
\int R_{\lambda}\left(\frac{1}{z-a_{\lambda}}, u\right) d z=\frac{g_{r-1}(u)}{\left(z-a_{\lambda}\right)^{r-1}}-\int\left\{\sum_{e} \frac{g_{r-1}^{\prime}(u) \cdot \phi_{e}(u)}{\left(z-a_{\lambda}\right)^{r-1}\left(z-a_{e}\right)}\right\} d z \\
+\int R^{\prime}\left(\frac{\dot{1}}{z-a_{\lambda}}, u\right) d z .
\end{aligned}
$$

Here, in the expression to the right, $\left(z-a_{\lambda}\right)^{r-1}$ is the highest power of $z-a_{\lambda}$ which appears in a denominator under the integral sign, and on reducing the order to which $u$ appears in the products $g_{r-1}^{\prime}(u) \cdot \phi_{e}(u)$ by aid of the equation $F(z, u)=0$ and on separating the factors $\left(z-a_{\lambda}\right)^{r-1}$ and $z-a_{e}$ from one another in the denominators by the method of partial fractions, we express our integral in the form:

$$
\begin{aligned}
\int R_{\lambda}\left(\frac{1}{z-a_{\lambda}}, u\right) d z= & \frac{g_{r-1}(u)}{\left(z-a_{\lambda}\right)^{r-1}}+\int R_{\lambda}^{\prime}\left(\frac{1}{z-a_{\lambda}}, u\right) d z \\
& +\sum_{e} \int S_{e}^{\prime}\left(\frac{1}{z-a_{e}}, u\right) d z+\int S_{0}^{\prime}(z, u) d z
\end{aligned}
$$


where $R_{\lambda}^{\prime}\left(1 /\left(z-a_{\lambda}\right), u\right)$ is a polynomial in $1 /\left(z-a_{\lambda}\right), u$ of degree $r-1$ in $1 /\left(z-a_{\lambda}\right), S_{e}^{\prime}\left(1 /\left(z-a_{e}\right), u\right)$ a polynomial in $1 /\left(z-a_{e}\right), u$ of degree 1 in $1 /\left(z-a_{e}\right)$, and $S_{0}^{\prime}(z, u)$ a polynomial in $z, u$, none of these polynomials hav. ing in $u$ a degree which is greater than $n-1$.

By the above process we have reduced by 1 the degree to which $1 /\left(z-a_{\lambda}\right)$ appears under the integral sign and by application of the same process to

$$
\int R_{\lambda}^{\prime}\left(\frac{1}{z-a_{\lambda}}, u\right) d z
$$

we should obtain a formula :

$$
\begin{aligned}
\int R_{\lambda}\left(\frac{1}{z-a_{\lambda}}, u\right) d z=\frac{g_{r-1}(u)}{\left(z-a_{\lambda}\right)^{r-1}} & +\frac{g_{r-2}(u)}{\left(z-a_{\lambda}\right)^{r-2}}+\int R_{\lambda}^{\prime \prime}\left(\frac{1}{z-a_{\lambda}}, u\right) d z \\
& +\sum_{e} \int S_{e}^{\prime \prime}\left(\frac{1}{z-a_{e}}, u\right) d z+\int S_{0}^{\prime \prime}(z, u) d z
\end{aligned}
$$

where $r-2$ is the degree of $R_{\lambda}^{\prime \prime}\left(1 /\left(z-a_{\lambda}\right), u\right)$ in $1 /\left(z-a_{\lambda}\right)$.

By successive applications of the process we should ultimately arrive at an expression for our integral in the form:

$$
\begin{aligned}
& \int R_{\lambda}\left(\frac{1}{z-a_{\lambda}}, u\right) d z=\frac{g_{r-1}(u)}{\left(z-a_{\lambda}\right)^{r-1}}+\frac{g_{r-2}(u)}{\left(z-a_{\lambda}\right)^{r-2}}+\cdots+\frac{g_{1}(u)}{z-a_{\lambda}} \\
& +\int \bar{R}_{\lambda}\left(\frac{1}{z-a_{\lambda}}, u\right) d z+\sum_{e} \int \bar{S}_{e}\left(\frac{1}{z-a_{e}}, u\right) d z+\int \bar{S}_{0}(z, u) d z,
\end{aligned}
$$

where $\bar{R}_{\lambda}\left(1 /\left(z-a_{\lambda}\right), u\right)$ is a polynomial in $1 /\left(z-a_{\lambda}\right), u$ of degree 1 in $1 /\left(z-a_{\lambda}\right), \bar{S}_{e}\left(1 /\left(z-a_{e}\right), u\right)$ a polynomial in $1 /\left(z-a_{e}\right), u$ of degree 1 in $1 /\left(z-a_{e}\right)$, and $\bar{S}_{0}(z, u)$ a polynomial in $z, u$.

\section{$\S 4$.}

We have obtained formula (13) on the assumption that no branch or double point corresponds to the value $z=a_{\lambda}$. Assuming on the contrary now that we have a branch or double point $\left(a_{\lambda}, b_{\lambda}\right)$ so that $z-a_{\lambda}$ is included among the factors $z-a_{\bullet}$, we derive from (11):

$$
\begin{array}{r}
\int R_{\lambda}\left(\frac{1}{z-a_{\lambda}}, u\right) d z=\frac{g_{r-1}(u)}{\left(z-a_{\lambda}\right)^{r-1}}+\int\left\{\frac{f_{r}(u)+(r-1) g_{r-1}(u)-g_{r-1}^{\prime}(u) \cdot \phi_{\lambda}(u)}{\left(z-a_{\lambda}\right)^{r}}\right. \\
\left.-\sum_{e}^{\prime} \frac{g_{r-1}^{\prime}(u) \cdot \phi_{e}(u)}{\left(z-a_{\lambda}\right)^{r-1}\left(z-a_{e}\right)}\right\} d z+\int R^{\prime}\left(\frac{1}{z-a_{\lambda}}, u\right) d z,
\end{array}
$$

where the accent in the sign of summation $\sum_{e}^{\prime}$ is intended to signify that the term corresponding to $a_{e}=a_{\lambda}$ is omitted from the summation. 
Here again we wish by a proper choice of $g_{r-1}(u)$ to rid ourselves of terms under the integral sign which involve $\left(z-a_{\lambda}\right)^{r}$ in their denominator.

For this it will be necessary that the expression

$$
f_{r}(u)+(r-1) g_{r-1}(u)-g_{r-1}^{\prime}(u) \cdot \phi_{\lambda}(u)
$$

be divisible by $z-a_{\lambda}$, after it has been reduced to the normal form in which $u$ does not appear to an order higher than $n-1$. Now if this expression in its reduced form is to be divisible by $z-a_{\lambda}$ it must, as it stands, be equal to 0 for each of the $n-1$ values $b_{\lambda}, b_{\lambda}^{\prime}, \ldots, b_{\lambda}^{(n-2)}$ of $u$ corresponding to the value $z=a_{\lambda}$, and must therefore be divisible by

It follows then that

$$
\phi_{\lambda}(u)=c_{\lambda}\left(u-b_{\lambda}\right)\left(u-b_{\lambda}^{\prime}\right) \cdots\left(u-b_{\lambda}^{(n-2)}\right)=\frac{c_{\lambda} F\left(a_{\lambda}, u\right)}{u-b_{\lambda}} .
$$

$$
f_{r}(u)+(r-1) g_{r-1}(u)
$$

also must be divisible by $\phi_{\lambda}(u)$, and since the degree of this expression in $u$ is $n-1$ we may write:

$$
f_{r}(u)+(r-1) g_{r-1}(u)=\kappa \phi_{\lambda}(u),
$$

where $\kappa$ is a constant which is yet to be determined.

For our function $g_{r-1}(u)$ we must therefore have

$$
g_{r-1}(u)=\frac{\kappa \phi_{\lambda}(u)-f_{r}(u)}{r-1},
$$

and on substituting this in (15) we obtain :

$$
\begin{aligned}
f_{r}(u)+(r-1) g_{r-1}(u)-g_{r-1}^{\prime}(u) \cdot \phi_{\lambda}(u) & =\left(\kappa-g_{r-1}^{\prime}(u)\right) \phi_{\lambda}(u) \\
& =\frac{1}{r-1}\left\{(r-1) \kappa-\kappa \phi_{\lambda}^{\prime}(u)+f_{r}^{\prime}(u)\right\} \phi_{\lambda}(u),
\end{aligned}
$$

which expression in its reduced form must be divisible by $z-a_{\lambda}$. Now we have :

$$
\left(u-b_{\lambda}\right) \phi_{\lambda}(u)=c_{\lambda} F\left(a_{\lambda}, u\right)=c_{\lambda}\left\{F\left(a_{\lambda}, u\right)-F(z, u)\right\},
$$

which is evidently divisible by $z-a_{\lambda}$. Also in order that the expression in (17) should be divisible by $\left(u-b_{\lambda}\right) \phi_{\lambda}(u)$ it is only necessary that the factor

$$
(r-1) \kappa-\kappa \phi_{\lambda}^{\prime}(u)+f_{r}^{\prime}(u)
$$

should be divisible by $u-b_{\lambda}$, which will be the case if we choose $\kappa$ so as to satisfy the condition

$$
\left\{(r-1)-\phi_{\lambda}^{\prime}\left(b_{\lambda}\right)\right\} \kappa+f_{r}^{\prime}\left(b_{\lambda}\right)=0 .
$$

On so choosing $\kappa$ the expression in (17) may be put into a form which is divisible by $z-a_{\lambda}$ and must therefore evidently also, in its reduced form, be 
divisible by this factor. Such a choice of $\kappa$ is of course always possible, excepting in the case where the coefficient of $\kappa$ in (18) is equal to 0 .

We have:

$$
\phi_{\lambda}^{\prime}\left(b_{\lambda}\right)=c_{\lambda}\left[\left(u-b_{\lambda}^{\prime}\right) \cdots\left(u-b_{\lambda}^{(n-2)}\right)\right]_{u=b_{\lambda}}=\frac{1}{2} c_{\lambda}\left[F_{u}^{\prime \prime}\left(a_{\lambda}, u\right)\right]_{u=b_{\lambda}} .
$$

It follows then that

and that we therefore have :

$$
\phi_{\lambda}^{\prime}\left(b_{\lambda}\right)=\frac{1}{2} \text { or } 1
$$

$$
\kappa=-\frac{f_{r}^{\prime}\left(b_{\lambda}\right)}{r-1-\phi_{\lambda}^{\prime}\left(b_{\lambda}\right)}=\frac{-f_{r}^{\prime}\left(b_{\lambda}\right)}{r-\frac{3}{2}} \text { or } \frac{-f_{r}^{\prime}\left(b_{\lambda}\right)}{r-2},
$$

according as $\left(a_{\lambda}, b_{\lambda}\right)$ is a branch or double point. The one case here excepted is evidently that in which we have to do with a double point $\left(a_{\lambda}, b_{\lambda}\right)$ and where at the same time $r=2$, in which case the coefficient of $\kappa$ in (18) is 0 . Substituting the values just obtained for $\kappa$ in the expression already given above for $g_{r-1}(u)$ we obtain :

$$
\begin{aligned}
g_{r-1}(u) & =-\left\{\frac{4 f_{r}^{\prime}\left(b_{\lambda}\right) \cdot \phi_{\lambda}(u)}{(2 r-2)(2 r-3)}+\frac{f_{r}(u)}{r-1}\right\} \\
& =-\left\{\frac{4}{(2 r-2)(2 r-3)} \frac{f_{r}^{\prime}\left(b_{\lambda}\right)}{F_{b_{\lambda}}^{\prime \prime}\left(a_{\lambda}, b_{\lambda}\right)} \frac{F\left(a_{\lambda}, u\right)}{u-b_{\lambda}}+\frac{f_{r}(u)}{r-1}\right\} \\
g_{r-1}(u) & =-\left\{\frac{f_{r}^{\prime}\left(b_{\lambda}\right) \cdot \phi_{\lambda}(u)}{(r-1)(r-2)}+\frac{f_{r}(u)}{r-1}\right\} \\
& =-\left\{\frac{2}{(r-1)(r-2)} \frac{f_{r}^{\prime}\left(b_{\lambda}\right)}{F_{b_{\lambda}}^{\prime \prime}\left(a_{\lambda}, b_{\lambda}\right)} \frac{F\left(a_{\lambda}, u\right)}{u-b_{\lambda}}+\frac{f_{r}(u)}{r-1}\right\},
\end{aligned}
$$

or

according as $\left(a_{\lambda}, b_{\lambda}\right)$ is a branch point or a double point, the exceptional case being here of course excluded.

The substitution of these expressions for $g_{r-1}(u)$ in (14) will enable us to rid ourselves of the $r$-th power of $z-a_{\lambda}$ in the denominator under the integral sign, the numerator

$$
f_{r}(u)+(r-1) g_{r-1}(u)-g_{r-1}^{\prime}(u) \cdot \phi_{\lambda}(u)
$$

in its reduced form being then divisible by $z-a_{\lambda}$.

By applying the principle of partial fractions to the terms of form

$$
\frac{g_{r-1}^{\prime}(u) \cdot \phi_{e}(u)}{\left(z-a_{\lambda}\right)^{r-1}\left(z-a_{e}\right)}
$$

we may, as in the section preceding, separate the factors $\left(z-a_{\lambda}\right)^{r-1}$ and $z-a_{e}$ in the denominators, and on reducing the powers of $u$ occurring in the various numerators we may put (14) into the form: 


$$
\begin{aligned}
\int R_{\lambda}\left(\frac{1}{z-a_{\lambda}}, u\right) d z= & \frac{g_{r-1}(u)}{\left(z-a_{\lambda}\right)^{r-1}}+\int R_{\lambda}^{\prime}\left(\frac{1}{z-a_{\lambda}}, u\right) d z \\
& +\sum_{e}^{\prime} \int S_{e}^{\prime}\left(\frac{1}{z-a_{e}}, u\right) d z+\int S_{0}^{\prime}(z, u) d z,
\end{aligned}
$$

where $R_{\lambda}^{\prime}\left(1 /\left(z-a_{\lambda}\right), u\right)$ is a polynomial in $1 /\left(z-a_{\lambda}\right), u$ of degree $r-1$ in $1 /\left(z-a_{\lambda}\right), S_{e}^{\prime}\left(1 /\left(z-a_{e}\right), u\right)$ a polynomial in $1 /\left(z-a_{e}\right), u$ of degree 1 in $1 /\left(z-a_{e}\right)$, and $S_{0}^{\prime}(z, u)$ a polynomial in $z, u$, the degrees in $u$ of these several polynomials being not greater than $n-1$.

By successive applications of the above process we shall evidently arrive at a reduced form,

$$
\begin{aligned}
& \int R_{\lambda}\left(\frac{1}{z-a_{\lambda}}, u\right) d z=\frac{g_{r-1}(u)}{\left(z-a_{\lambda}\right)^{r-1}}+\frac{g_{r-2}(u)}{\left(z-a_{\lambda}\right)^{r-2}}+\cdots+\frac{g_{1}(u)}{z-a_{\lambda}} \\
& \quad+\int \bar{R}_{\lambda}\left(\frac{1}{z-a_{\lambda}}, u\right) d z+\sum_{e} \int \bar{S}_{e}\left(\frac{1}{z-a_{e}}, u\right) d z+\int \bar{S}_{0}(z, u) d z,
\end{aligned}
$$

which is a form of the same type as that obtained in (13) for the case of the section preceding, excepting that here $1_{i}^{\prime}\left(z-a_{\lambda}\right)$ may present itself to the second power in the polynomial $\bar{R}_{\lambda}\left(1 /\left(z-a_{\lambda}\right), u\right)$ where to $z=a_{\lambda}$ corresponds a double point.

We shall now consider more in detail the special case $r=2$ in which we have failed to get rid of the power $1 /\left(z-a_{\lambda}\right)^{2}$ under the sign of integration.

The difficulty here arose in connection with the equation (18) which takes the form

$$
0 \cdot \kappa+f_{2}^{\prime}\left(b_{\lambda}\right)=0,
$$

when $r=2$ and when at the same time $\left(a_{\lambda}, b_{\lambda}\right)$ is a double point. This equation cannot be satisfied by any finite value of $\kappa$ unless we happen to have $f_{2}^{\prime}\left(b_{\lambda}\right)=0$, in which case $\kappa$ may have any arbitrary value. Supposing then that we have to do with this case we shall take $\kappa=0$ when from (16) we obtain

$$
g_{r-1}(u)=-\frac{f_{r}(u)}{r-1}
$$

and the expression in (17) becomes

$$
\frac{1}{r-1} f_{r}^{\prime}(u) \cdot \phi_{\lambda}(u)
$$

which is divisible by $\left(u-b_{\lambda}\right) \phi_{\lambda}(u)$ and in its reduced form is therefore divisible by $z-a_{\lambda}$.

The numerator of $\left(z-a_{\lambda}\right)^{r}=\left(z-a_{\lambda}\right)^{2}$ in (14) is then divisible by $z-a_{\lambda}$ and $1 /\left(z-a_{\lambda}\right)$ will therefore appear only to the first power under the sign of integration. 
If however we have $f_{2}^{\prime}\left(b_{\lambda}\right) \neq 0$, I shall define a function $\bar{f}_{2}(u)$ by the equation

$$
f_{2}(u)=\bar{f}_{2}(u)+\bar{h} \phi_{\lambda}(u) \text {, }
$$

where $\bar{h}=f_{2}^{\prime}\left(b_{\lambda}\right)$.

Since $\phi_{\lambda}^{\prime}\left(b_{\lambda}\right)=1$, it follows then that $\bar{f}_{2}^{\prime}\left(b_{\lambda}\right)=0$.

On writing $r=2$ in (11) we shall have:

$$
\begin{aligned}
\int \bar{R}_{\lambda}\left(\frac{1}{z-a_{\lambda}}, u\right) d z & =\int \frac{f_{2}(u)}{\left(z-a_{\lambda}\right)^{2}} d z+\int R^{\prime}\left(\frac{1}{z-a_{\lambda}}, u\right) d z \\
& =\int \frac{\overline{f_{2}}(u)}{\left(z-a_{\lambda}\right)^{2}} d z+\int \frac{\bar{h} \phi_{\lambda}(u)}{\left(z-a_{\lambda}\right)^{2}} d z+\int R^{\prime}\left(\frac{1}{z-a_{\lambda}}, u\right) d z \\
& =\int \frac{\overline{f_{2}}(u)}{\left(z-a_{\lambda}\right)^{2}} d z+\int \frac{h F\left(a_{\lambda}, u\right)}{\left(z-a_{\lambda}\right)^{2}\left(u-b_{\lambda}\right)} d z \\
& +\int R^{\prime}\left(\frac{1}{z-a_{\lambda}}, u\right) d z
\end{aligned}
$$

Apart from the second integral on the right the expression here appearing is of the type just considered, in which we saw that we could get rid of the second power of $1 /\left(z-a_{\lambda}\right)$ under the sign of integration.

Hence in the case where to $z=a_{\lambda}$ corresponds a double point we may evidently replace $(20)$ by a formula of the type :

$$
\begin{aligned}
& \int R_{\lambda}\left(\frac{1}{z-a_{\lambda}}, u\right) d z=\frac{g_{r-1}(u)}{\left(z-a_{\lambda}\right)^{r-1}}+\frac{g_{r-2}(u)}{\left(z-a_{\lambda}\right)^{r-2}}+\cdots+\frac{g_{1}(u)}{z-a_{\lambda}} \\
+ & h_{\lambda} \int \frac{F\left(a_{\lambda}, u\right.}{\left(z-a_{\lambda}\right)^{2}\left(u-b_{\lambda}\right)} d z+\sum_{e} \int \bar{S}_{e}\left(\frac{1}{z-a_{e}}, u\right) d z+\int \bar{S}_{0}(z, u) d z
\end{aligned}
$$

and under this formula, on putting 0 for the constant coefficient $h_{\lambda}$, may be supposed to be included that also for the case in which a branch point corresponds to $z=a_{\lambda}$, for in this case the integral

$$
\int \bar{R}_{\lambda}\left(\frac{1}{z-a_{\lambda}}, u\right) d z
$$

in (20) may be supposed to be included under the summation

$$
\sum_{e} \int \bar{S}\left(\frac{1}{z-a_{e}}, u\right) d z
$$

the first power only of $1 /\left(z-a_{\lambda}\right)$ here presenting itself under the sign of integration. 
$\S 5$.

By the processes of the two preceding sections, therefore, we can reduce any integral

$$
\int R_{\lambda}\left(\frac{1}{z-a_{\lambda}}, u\right) d z
$$

to the form given on the right of (21) or (13) respectively, according as a singular point does or does not correspond to the value $z=a_{\lambda}$. After the substitution of the reduced forms so obtained for the several integrals in the summation to the right of (4) this equation will assume the form :

$$
\begin{array}{r}
\int R(z, u) d z=P(z, u)+\sum_{\lambda} \int \frac{h_{\lambda} F\left(a_{\lambda}, u\right)}{\left(z-a_{\lambda}\right)^{2}\left(u-b_{\lambda}\right)} d z+\sum_{\lambda} \int \bar{R}_{\lambda}\left(\frac{1}{z-a_{\lambda}}, u\right) d z \\
\quad+\sum_{e} \int S_{e}^{\prime \prime}\left(\frac{1}{z-a_{e}}, u\right) d z+\int S_{0}^{\prime \prime}(z, u) d z,
\end{array}
$$

where the first summation on the right-hand side is supposed to extend to the double points $\left(a_{\lambda}, b_{\lambda}\right)$ only, the second summation to those values of $a_{\lambda}$ for which negative powers of $z-a_{\lambda}$ occur in $R(z, u)$, from which however we may exclude elements corresponding to branch or double points, and the third summation to those values $a_{e}$ to which correspond branch or double points.

The last two summations we may fuse into one, reducing the above formula to the form :

$$
\begin{aligned}
& \int R(z, u) d z=P(z, u)+\sum_{\lambda} \int \frac{h_{\lambda} F\left(a_{\lambda}, u\right)}{\left(z-a_{\lambda}\right)^{2}\left(u-b_{\lambda}\right)} d z \\
& \quad+\sum_{\lambda} \int S_{\lambda}^{\prime \prime}\left(\frac{1}{z-a_{\lambda}}, u\right) d z+\int S_{0}^{\prime \prime}(z, u) d z,
\end{aligned}
$$

where the first summation on the right still extends to the double points alone while the second summation extends to those values of $a_{\lambda}$ to which correspond branch or double points and over and above these to all those values for which negative powers of the corresponding factor $z-a_{\lambda}$ appear in the expression of the function $R(z, u)$.

The function

$$
\frac{F\left(a_{\lambda}, u\right)}{\left(z-a_{\lambda}\right)^{2}\left(u-b_{\lambda}\right)}
$$

becomes infinite to the first order for each of the $n-1$ points corresponding to the value $z=a_{\lambda}$ and it is evident in fact that for a finite value of $z$ none of the functions under the sign of integration to the right of (22) becomes infinite to an order greater than 1.* Apart from the points at $\infty$, therefore, the integrals

* While employing the form of expression "infinity of the first, second, etc., order" in the accepted sense of RIEMANN, I shall find it convenient for the temporary purpose of this paper to 
on the right of $(22)$ can only become infinite logarithmically. The residues corresponding to the several infinities are immediately determinable and we may suppose the logarithmic portion of the expression in (22) to be represented by an integral of the third kind which we shall indicate by (III) and which is made up of a sum with constant coefficients of a number of elementary integrals of the third kind.

Formula (22) may then be reduced to the form

$$
\int R(z, u) d z=P(z, u)+\mathrm{III}+\int H(z, u) d z,
$$

where the integral $\int H(z, u) d z$ does not become infinite for any finite value of $z$. The function $H(z, u)$ itself will then not become infinite to an order as great as $\mathbf{1}$ for any finite value of the variable. What the form of our function $H(z, u)$ is would appear immediately from the forms of the expressions under the signs of integration to the right of $(22)$.

We shall find it convenient however to here determine directly once for all the general form of a rational function $H(z, u)$ which does not become infinite to an order as great as 1 for a finite value of $z$.

As in (3), by the application of the principle of partial fractions, we may suppose our function to be expressed as a sum in the form

$$
H(z, u)=\sum_{\lambda} H_{\lambda}\left(\frac{1}{z-a_{\lambda}}, u\right)+H_{0}(z, u) .
$$

The function $H(z, u)$ will or will not be infinite for a point corresponding to the value $z=a_{\lambda}$ according as this is or is not the case for the corresponding element $H_{\lambda}\left(1 /\left(z-a_{\lambda}\right), u\right)$ on the right. Writing the element in the form

$$
H_{\lambda}\left(\frac{1}{z-a_{\lambda}}, u\right)=\frac{\sigma_{1}(u)}{z-a_{\lambda}}+\cdots+\frac{\sigma_{s-1}(u)}{\left(z-a_{\lambda}\right)^{s-1}}+\frac{\sigma_{s}(u)}{\left(z-a_{\lambda}\right)^{s}},
$$

where the numerators $\sigma(u)$ are polynomials in $u$ of degree $n-1$, we see that it will become infinite to the order $s$ for some point corresponding to the value $z=a_{\lambda}$ unless we have

$$
\sigma_{s}(u)=0
$$

for each of the values $u$ corresponding to $z=a_{\lambda}$. The number of these values therefore cannot be greater than $n-1$.

It follows then that in (24) the summation may be confined to values $a_{\lambda}$ to which correspond branch or double points. Since for each of the $n-1$ values of $u$ corresponding to $z=a_{\lambda}$ we have $\sigma_{\lrcorner}(u)=0$, we may write

make use of the expression "infinite to the order $\frac{1}{2}, 1$, etc.," to denote an infinity of the character indicated by $(z-a)^{-\frac{1}{2}},(z-a)^{-1}$, etc. The two forms of expression then differ in their signification when employed relatively to a branch point, but it is hoped that this word of explanation will prevent any confusion from arising in the mind of the reader. 
where $h$ is a constant.

$$
\sigma_{\triangleleft}(u)=\frac{h F\left(a_{\lambda}, u\right)}{u-b_{\lambda}}
$$

In the case of a branch point $\left(a_{\lambda}, b_{\lambda}\right)$ the function

$$
\frac{\sigma_{s}(u)}{\left(z-a_{\lambda}\right)^{s}}=\frac{h F\left(a_{\lambda}, u\right)}{\left(z-a_{\lambda}\right)^{s}\left(u-b_{\lambda}\right)},
$$

and therewith the element $H_{\lambda}$, here in question also, would evidently be infinite to the order $s-\frac{1}{2}$ so that we cannot have $s \equiv 2$. It follows therefore that $s=1$.

In the case where a branch point corresponds to the value $z=a_{\lambda}$ then we shall have for our element:

$$
H_{\lambda}\left(\frac{1}{z-a_{\lambda}}, u\right)=\frac{h F\left(a_{\lambda}, u\right)}{\left(z-a_{\lambda}\right)\left(u-b_{\lambda}\right)},
$$

where $h$ may have any arbitrary constant value. In the case where to $z=a_{\lambda}$ corresponds a double point let us suppose, if possible, that we have $s \equiv 2$. In this case the expression

$$
\frac{\sigma_{n-1}(u)}{\left(z-a_{\lambda}\right)^{s-1}}+\frac{\sigma_{s}(u)}{\left(z-a_{\lambda}\right)^{s}}
$$

must evidently not be infinite to an order greater than $s-2$, for then it would be infinite to the order 1 at least, and this would hold also in regard to the element on the left of (25). The expression

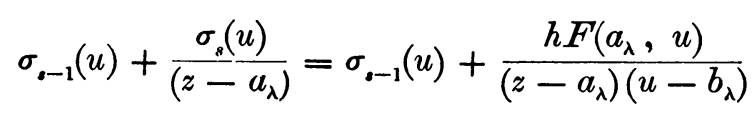

must therefore vanish for each of the $n-1$ points corresponding to the value $z=a_{\lambda}$ and in particular for each of the branches through the double point $\left(a_{\lambda}, b_{\lambda}\right)$.

On indicating the directions of the tangents at the double point by $m_{1}$ and $m_{2}$ respectively, we must then have :

and therefore

$$
\begin{aligned}
& \sigma_{s-1}\left(b_{\lambda}\right)+\frac{1}{2} h m_{1}\left[F_{u}^{\prime \prime}\left(a_{\lambda}, u\right)\right]_{u=b_{\lambda}}=0, \\
& \sigma_{s-1}\left(b_{\lambda}\right)+\frac{1}{2} h m_{2}\left[F_{u}^{\prime \prime}\left(a_{\lambda}, u\right)\right]_{u=b_{\lambda}}=0,
\end{aligned}
$$

$$
h\left(m_{1}-m_{2}\right)\left[F_{u}^{\prime \prime}\left(a_{\lambda}, u\right)\right]_{u=b_{\lambda}}=0 .
$$

Since, however, $m_{1} \neq m_{2}$ and $\left[F_{u}^{\prime \prime}\left(a_{\lambda}, u\right)\right]_{u=b_{\lambda}} \neq 0$, this is impossible unless we have $h=0$ and consequently $\sigma_{s}(u)=0$.

It follows therefore that we cannot have $s \equiv 2$.

For $s=1$ we shall have 


$$
\frac{\sigma_{1}(u)}{z-a_{\lambda}}=\frac{h F\left(a_{\lambda}, u\right)}{\left(z-a_{\lambda}\right)\left(u-b_{\lambda}\right)}
$$

a function which is finite for all the $n-1$ points corresponding to $z=a_{\lambda}$.

In the case where a double point corresponds to the value $z=a_{\lambda}$, as in the case of a branch point, the element in (25) takes the form given in (26), and for our function $H(z, u)$ in (24) we may write

$$
H(z, u)=\sum_{e} \frac{h_{e} F\left(a_{e}, u\right)}{\left(z-a_{e}\right)\left(u-b_{e}\right)}+H_{0}(z, u),
$$

where the summation is supposed to extend to all those values $a_{e}$ to which correspond branch or double points, where the coefficients $h_{e}$ may have any arbitrary constant values, and where $H_{0}(z, u)$ is an arbitrary polynomial in $z, u$. This then represents the most general function $\dot{H}(z, u)$ which does not become infinite to an order as great as 1 for any finite value of $z$.

The most general function of $z, u$ which becomes infinite only at $\infty$ we should evidently derive from (27) on equating to 0 therein all those coefficients $h_{e}$ corresponding to the branch points, or in other words, on supposing the summation in (27) to be extended only to those values $a_{e}$ to which correspond double points.

Returning now to formula (23) we may by (27) express the same in the form :

$$
\int R(z, u) d z=P(z, u)+\mathrm{III}+\int\left\{\sum_{e} \frac{h_{e} F^{\prime}\left(a_{e}, u\right)}{\left(z-a_{e}\right)\left(u-b_{e}\right)}+H_{0}(z, u)\right\} d z
$$

$\S 6$.

We shall now occupy ourselves with the reduction of the integral

$$
\int H_{0}(z, u) d z \text {. }
$$

Supposing the degree of the polynomial $H_{0}(z, u)$ in $z, u$ to be $n+r-1$ where $r \equiv 0$, we shall indicate this by the suffix $n+r-1$ and write

$$
H_{0}(z, u)=T_{n+r-1}(z, u) \text {. }
$$

We may evidently express $T_{n+r-1}(z, u)$ in the form

$$
T_{n+r-1}(z, u)=z^{r} t(z, u)+T_{n+r-2}^{\prime}(z, u),
$$

where the function $t(z, u)$ is a homogeneous polynomial in $z, u$ of degree $n-1$ and where $T_{n+r-2}^{\prime}(z, u)$ is a polynomial in $z ; u$ in general non-homogeneous and of degree $n+r-2$.

It is evident that the function $T_{n+r-1}(z, u)$ cannot be infinite to an order higher than $n+r-1$ for any of the $n$ branches at $\infty$, and that it must be infinite to this order for one at least of these branches we may readily show. 
Namely, on substituting in (29) for $u$ in terms of $z$ from the equation to a given one of the branches at $\infty$, the coefficient of $z^{n+r-1}$ will be furnished by the element $z^{r} t(z, u)$ and will be the same as that obtained on substituting $u=\kappa z$ from the corresponding equation in (7). The term in $z^{n+r-1}$ so obtained is

$$
z^{r} t(z, \kappa z)=z^{n+r-1} t(1, \kappa),
$$

and the coefficient $t(1, \kappa)$ of $z^{n+r-1}$ will be different from 0 in the case of one at least of the $n$ branches, as otherwise we should have

$$
t(1, \kappa)=0
$$

for each of the $n$ values $\kappa_{1}, \kappa_{2}, \ldots, \kappa_{n}$ and this equation would therefore be an identity since its degree in $\kappa$ is $n-1$. It would follow then also that we must have

$$
t(\dot{z}, u)=0
$$

identically, which however is not consistent with our hypothesis that our function $T_{n+r-1}(z, u)$ has the degree $n+r-1$. We conclude then that a polynomial $T_{n+r-1}(z, u)$ becomes infinite to the order $n+r-1$, that is, to an order equal to its degree, for ore or more of the branches at $\infty$.

Now consider the integral

$$
\int z^{r} t\left(z, v_{i}\right) d z=\int\left\{z^{r} t(z, u)-\frac{d}{d z} \frac{z^{r+1} t(z, u)}{n+r}\right\} d z+\frac{z^{r+1} t(z, u)}{n+r} .
$$

The expression under the integral sign to the right of this identity evidently does not become infinite to the order $n+r-1$ for any of the branches at $\infty$. It may be written in the form :

$$
\begin{aligned}
z^{r} t(z, u)-\frac{d}{d z} \frac{z^{r+1} t(z, u)}{n+r} & =\frac{(n-1) z^{r} t(z, u)}{n+r}-\frac{z^{r+1}}{n+r}\left(\frac{\partial t}{\partial z}+\frac{\partial t}{\partial u} \frac{d u}{d z}\right) \\
& =\frac{(n-1) z^{-t} t(z, u)}{n+r}-\frac{z^{r+1}}{n+r}\left(\frac{\partial t}{\partial z}+\frac{\partial t}{\partial u} \sum_{e} \frac{\phi_{e}(u)}{z-a_{e}}\right),
\end{aligned}
$$

where for $d u / d z$ we have substituted its expression given in (5). From the last written form we immediately derive:

$$
z^{r} t(z, u)-\frac{d}{d z} \frac{z^{r+1} t(z, u)}{n+r}=T^{\prime \prime}(z, u)+\sum_{e} \frac{\theta_{e}(u)}{z-a_{e}},
$$

where $T^{\prime \prime}(z, u)$ is a polynomial in $(z, u)$ of degree in $u$ not greater than $n-1$ and where the functions $\theta_{e}(u)$ are polynomials in $u$ alone whose degrees are equal to or less than $n-1$. 
Further, the expression to the left of the formula just written, as we have seen above, does not become infinite to the order $n+r-1$ for any of the branches at $\infty$ and as none of the elements in the summation

$$
\sum_{e} \frac{\theta_{e}(u)}{z-a_{e}}
$$

on the right becomes infinite to an order which is greater than $n-2$, it follows that the polynomial $T^{\prime \prime}(z, u)$ also does not become infinite to the order $n+r-1$ and that its degree in $z, u$ therefore is not greater than $n+r-2$. Indicating this fact by the suffix $n+r-2$, we may write:

$$
z^{r} t(z, u)-\frac{d}{d z} \frac{z^{r+1} t(z, u)}{n+r}=T_{n+r-2}^{\prime \prime}(z, u)+\sum_{e} \frac{\theta_{e}(u)}{z-a_{e}} ;
$$

and substituting the form so obtained for the function under the integral sign to the right of $(30)$ we derive :

$$
\int z^{r} t(z, u) d z=\int\left\{T_{n+r-2}^{\prime \prime}(z, u)+\sum_{e} \frac{\theta_{e}(u)}{z-a_{e}}\right\} d z+\frac{z^{r+1} t(z, u)}{n+r} .
$$

Combining this result with (29) we obtain :

$$
\begin{array}{r}
\int T_{n+r-1}(z, u) d z=\int\left\{T_{n+r-2}^{\prime}(z, u)+T_{n+r-2}^{\prime \prime}(z, u)+\sum_{e} \frac{\theta_{e}(u)}{z-a_{e}}\right\} d z \\
+\frac{z^{r+1} t(z, u)}{n+r},
\end{array}
$$

and this we may more concisely write in the form

$$
\int T_{n+r-1}(z, u) d z=g_{n+r}(z, u)+\sum_{e} \int \frac{\theta_{e}(u)}{z-a_{e}} d z+\int T_{n+r-2}(z, u) d z,
$$

where

$$
g_{n+r}(z, u)=\frac{z^{r+1} t(z, u)}{n+r}, \quad T_{n+r-2}(z, u)=T_{n+r-2}^{\prime}(z, u)+T_{n+r-2}^{\prime \prime}(z, u) .
$$

Repeated application of the above process of reduction gives rise to a succession of integrals

$$
\int T_{n+r-2}(z, u) d z, \quad \int T_{n+r-3}(z, u) d z, \cdots, \int T_{n-2}(z, u) d z,
$$

and enables us to reduce our integral to an expression of the form

$$
\int T_{n+r-1}(z, u) d z=G_{n+r}(z, u)+\sum_{e} \int \frac{\sigma_{e}(u)}{z-a_{s}} d z+\int T_{n-2}(z, u) d z,
$$

where $G_{n+r}(z, u)$ and $T_{n-2}(z, u)$ are polynomials in $z, u$ of degrees $n+r$ and $n-2$ respectively, and where the functions $\sigma_{e}(u)$ are polynomials in $u$ of degree $n-1$. 
From the manner in which the functions

$$
\frac{\sigma_{e}(u)}{z-a_{e}}
$$

arise in the process above it is further self-evident that they do not become infinite to an order as great as $\mathbf{1}$ for the values $a_{e}$, so that their sum will constitute a function of the type represented in (27) and may be expressed in the form :

$$
\sum_{e} \frac{\sigma_{e}(u)}{z-a_{e}}=\sum_{e} \frac{a_{e} F\left(a_{e}, u\right)}{\left(z-a_{e}\right)\left(u-b_{e}\right)},
$$

where the coefficients $a_{e}$ are constants and the summation is supposed to extend to those values $a_{e}$ to which correspond branch or double points.

For formula (33) we may therefore write :

$$
\int T_{n+r-1}(z, u) d z=G_{n+r}(z, u)+\sum_{e} \int \frac{a_{e} F\left(a_{e}, u\right)}{\left(z-a_{e}\right)\left(u-b_{e}\right)} d z+\int T_{n-2}(z, u) d z
$$

and on substituting for $\int H_{0}(z, u) d z=\int T_{n+r-1}(z, u) d z$ in (28) the expression here obtained, we arrive at a reduced form for our original integral $\int R(z, u) d z$ which may be written :

$$
\int R(z, u) d z=\bar{G}(z, u)+\mathrm{III}+\int\left\{\sum_{e} \frac{\beta_{e} F\left(a_{e}, u\right)}{\left(z-a_{e}\right)\left(u-b_{e}\right)}+T_{n-2}(z, u)\right\} d z,
$$

where III is an integral of the third kind, $G(z, u)$ a rational function of $z, u$, $T_{n-2}(z, u)$ a polynomial in $z, u$ of degree $n-2$ and the $\beta_{e}$ 's constants, the summation with regard to $e$ being extended to all the branch and double points of our curve.

\section{$\S 7$.}

Our reductions of the integral $\int R(z, u) d z$ in the above have so far consisted in getting rid of various infinities of the function under the sign of integration

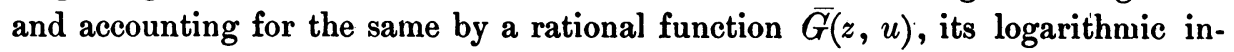
finities at the same time being separated from the main integral and incorporated in an integral of the third kind III.

We shall now consider the remaining integral to the right of (35) with a view to a further reduction. The expression under the integral sign is of the type represented in (27), which for no finite value of $z$ becomes infinite to an order which is as great as 1 . The integral itself,

$$
\int\left\{\sum_{e} \frac{\beta_{e} F\left(a_{e}, u\right)}{\left(z-a_{e}\right)\left(u-b_{e}\right)}+T_{n-2}(z, u)\right\} d z,
$$

will therefore be finite for all finite values of $z$ and for $z=\infty$ will not become 
infinite to an order which is greater than $n-1$ since the function under the sign of integration is evidently not infinite to an order which is greater than $n-2$ for any of the points at $\infty$. If now we would attempt a further elinination of infinities from under the sign of integration by the aid of a rational function of $z, u$ and that without introducing any new infinities, the most general function at our disposal is included under the type (27) and will evidently have the form

$$
\sum_{e} \frac{\gamma_{e} F\left(a_{e}, u\right)}{\left(z-a_{e}\right)\left(u-b_{e}\right)}+T_{n-1}(z, u),
$$

where $T_{n-1}(z, u)$ is a polynomial in $z, u$ of degree $n-1$, the summation with regard to $e$ being supposed to extend to all the double points and the coefficients $\gamma_{e}$ having any arbitrary values; for this is the most general rational function whose infinities are all at $\infty$ and which does not there become infinite to an order which is greater than $n-1$.

The number of coefficients which the polynomial $T_{n-1}(z, u)$ places at our disposal is $\frac{1}{2} n(n+1)$, or we may say $\frac{1}{2} n(n+1)-1$ on rejecting the constant term which could only serve to modify the arbitrary constant of integration in (36), and the number of the coefficients $\gamma_{e}$ in the expression (37) is $d$, the number of the double points of our curve. In all, then, the number of undetermined coefficients which the expression (37) places at our disposal is

$$
d+\frac{1}{2} n(n+1)-1 \text {. }
$$

Now the integral in (36) will evidently in general become infinite to the order $n-1$ for each of the $n$ branches at $\infty$, possessing therefore in all $n(n-1)$ infinities all situated at $\infty$. If then each of these infinities requires an extra available constant in order to eliminate it from under the sign of integration, the number of available constants placed at our disposal by the expressions (37) will fall short of that requisite by at least

that is, by at least

$$
n(n-1)-d-\frac{1}{2} n(n+1)+1 \text {, }
$$

$$
p=\frac{1}{2}(n-1)(n-2)-d .
$$

In order to make good this deficit I shall introduce $p$ more constants, introducing however with each one of them at the same time a new infinity. Namely instead of (37) I shall consider the expression

$$
\sum_{\lambda=1}^{d+p} \frac{\gamma_{\lambda} F\left(a_{\lambda}, u\right)}{\left(z-a_{\lambda}\right)\left(u-b_{\lambda}\right)}+T_{n-1}(z, u)
$$

in which the summation is supposed to extend not only to the double points, but also to $p$ additional points $\left(a_{\lambda}, b_{\lambda}\right)$ which do not coincide with double points but may otherwise be regarded as arbitrarily chosen. For these $p$ points, 
in case the corresponding coefficients $\gamma$ are not zero, the function in (38) will evidently become infinite to the first order. For all other finite points it will be finite and for the $n$ points at $\infty$ it will in general become infinite to the order $n-1$.

In the sequel we shall find that the $n(n-1)$ constants placed at our disposal by the expression (38) are all available and none of them superfluous for the purpose of ridding ourselves of all the infinities at $\infty$ of the general integral of the type $(36)$.

Consider the expression

$$
\begin{aligned}
\int\left\{\sum_{e} \frac{\beta_{e} F\left(a_{e}, u\right)}{\left(z-a_{e}\right)\left(u-b_{e}\right)}+T_{n-2}(z, u)\right\} d z & \\
& -\left\{\sum_{\lambda=1}^{d+p} \frac{\gamma_{\lambda} F\left(a_{\lambda}, u\right)}{\left(z-a_{\lambda}\right)\left(u-b_{\lambda}\right)}+T_{n-1}(z, u)\right\},
\end{aligned}
$$

obtained on subtracting the function in (38) from the integral in (36). Regarding the function under the sign of integration as given and the coefficients in the subtracted function as undetermined, we shall attempt to determine the latter so that the expression in (39) may have no infinities at $\infty$.

From the equation to our curve in (1) we have :

whence

$$
\begin{aligned}
\frac{F\left(a_{\lambda}, u\right)}{\left(z-a_{\lambda}\right)\left(u-b_{\lambda}\right)} & =\frac{F\left(a_{\lambda}, u\right)-F\left(a_{\lambda}, b_{\lambda}\right)}{\left(z-a_{\lambda}\right)\left(u-b_{\lambda}\right)}=\frac{\sum_{r s} e_{r s} a_{\lambda}^{r} u^{s}-\sum_{r s} e_{r s} a_{\lambda}^{r} b_{\lambda}^{s}}{\left(z-a_{\lambda}\right)\left(u-b_{\lambda}\right)} \\
& =\frac{\sum_{r s} e_{r s} a_{\lambda}^{r}\left(u^{s}-b_{\lambda}^{s}\right)}{\left(z-a_{\lambda}\right)\left(u-b_{\lambda}\right)},
\end{aligned}
$$

$$
\begin{aligned}
\frac{F\left(a_{\lambda}, u\right)}{\left(z-a_{\lambda}\right)\left(u-b_{\lambda}\right)} & =\left(z-a_{\lambda}\right)^{-1} \sum_{r s} e_{r s} a_{\lambda}^{r}\left(u^{s-1}+u^{s-2} b_{\lambda}+\cdots+b_{\lambda}^{s-1}\right) \\
& =\left(z-a_{\lambda}\right)^{-1} \sum_{s=1}^{n}\left\{a_{\lambda}, b_{\lambda}\right\}_{\ominus} u^{n-s},
\end{aligned}
$$

where

We may then write :

$$
\left\{a_{\lambda}, b_{\lambda}\right\}_{\odot}=\sum_{\sigma=0}^{\delta-1} \sum_{\rho=0}^{\sigma} e_{\rho, n-\sigma} a_{\lambda}^{\rho} b_{\lambda}^{s-\sigma-1} .
$$

$$
\begin{aligned}
\frac{F\left(a_{\lambda}, u\right)}{\left(z-a_{\lambda}\right)\left(u-b_{\lambda}\right)} & =\left\{\frac{1}{z}+\frac{a_{\lambda}}{z^{2}}+\cdots+\frac{a_{\lambda}^{n-2}}{z^{n-1}}+\frac{a_{\lambda}^{n-1}}{z^{n-1}\left(z-a_{\lambda}\right)}\right\} \sum_{s=1}^{n}\left\{a_{\lambda}, b_{\lambda}\right\}_{\bullet} u^{n-s} \\
& =\left\{\frac{1}{z}+\frac{a_{\lambda}}{z^{2}}+\cdots+\frac{a_{\lambda}^{n-2}}{z^{n-1}}\right\} \sum_{s=1}^{n}\left\{a_{\lambda}, b_{\lambda}\right\}_{\bullet} u^{n-s}+P_{\lambda}(z, u),
\end{aligned}
$$


where $P_{\lambda}(z, u)$ is a rational function of $z, u$ which is equal to 0 for $z=\infty$. This we may evidently further reduce to the form:

$$
\frac{F\left(a_{\lambda}, u\right)}{\left(z-a_{\lambda}\right)\left(u-b_{\lambda}\right)}=\sum_{s=1}^{n-1} \sum_{r=1}^{n-s} a_{\lambda}^{r-1}\left\{a_{\lambda}, b_{\lambda}\right\}_{\diamond} z^{-r} u^{n-s}+\bar{P}_{\lambda}(z, u)
$$

where $\bar{P}_{\lambda}(z, u)$ is a rational function of $z, u$ which is equal to 0 for $z=\infty$. The second of the two summations in (39) may then be written in the form:

$$
\begin{aligned}
\sum_{\lambda=1}^{d+p} \frac{\gamma_{\lambda} F\left(a_{\lambda}, u\right)}{\left(z-a_{\lambda}\right)\left(u-b_{\lambda}\right)} & =\sum_{\lambda=1}^{d+p} \sum_{s=1}^{n-1} \sum_{r=1}^{n-s} \gamma_{\lambda} a_{\lambda}^{r-1}\left\{a_{\lambda}, b_{\lambda}\right\}_{\triangleleft} z^{-r} u^{n-s}+\bar{P}(z, u) \\
& =\sum_{s=1}^{n-1} \sum_{r=1}^{n-s} c_{n-s-r, n-s} z^{-r} u^{n-s}+\bar{P}(z, u) \\
& =\sum_{s=1}^{n-2} \sum_{r=1}^{n-s-1} c_{n-s-r, n-s} z^{-r} u^{n-s}+P(z, u),
\end{aligned}
$$

where $\bar{P}(z, u)$ and $P(z, u)$ are rational functions of $z, u$ which are finite for $z=\infty$, the former function in particular having the value 0 for this value of the variable, and where for the coefficients $c$ we have

$$
c_{n-s-r, n-s}=\sum_{\lambda=1}^{d+p} \gamma_{\lambda} a_{\lambda}^{r-1}\left\{a_{\lambda}, b_{\lambda}\right\}_{\diamond} \cdot
$$

For the suffixes of the coefficients $c$ we shall find it convenient to choose, as we have done here, the sum of the exponents of $z$ and $u$ and the exponent of $u$ respectively.

On writing

$$
\gamma_{i \kappa}=\sum_{\lambda=1}^{d+p} \gamma_{\lambda} a_{\lambda}^{i} b_{\lambda}^{\kappa}
$$

we shall evidently have

$$
c_{n-s-r, n-s}=\sum_{\sigma=0}^{s-1} \sum_{\rho=0}^{\sigma} e_{\rho, n-\sigma} \gamma_{r-1+\rho, s-1-\sigma} .
$$

Since the exponents of $z$ which appear in the summation to the right of (42) are all negative whereas in the polynomial $T_{n-1}(z, u)$ in (39) no such exponents make their appearance, it will not be inconsistent with the notation employed above if we write:

$$
T_{n-1}(z, u)=\sum_{s=1}^{n} \sum_{r=0}^{s-1} c_{n-s+r, n-s} z^{r} u^{n-s}
$$


Combining (42) and (45) we have :

$$
\begin{aligned}
T_{n-1}(z, u)+\sum_{\lambda=1}^{d+p} \frac{\gamma_{\lambda} F\left(a_{\lambda}, u\right)}{\left(z-a_{\lambda}\right)\left(u-b_{\lambda}\right)}= & \sum_{s=1}^{n} \sum_{r=0}^{s-1} c_{n-s+r, n-s} z^{r} u^{n-s} \\
& +\sum_{s=1}^{n-1} \sum_{r=1}^{n-s} c_{n-s-r, n-s} z^{-r} u^{n-s}+\bar{P}(z, u) \\
= & \sum_{s=1}^{n} \sum_{r=-(n-s)}^{s-1} c_{n-s+r, n-s} z^{r} u^{n-s}+\bar{P}(z, u) .
\end{aligned}
$$

This again we may write in the form:

where

$$
\begin{aligned}
\sum_{\lambda=1}^{d+p} \frac{\gamma_{\lambda} F\left(a_{\lambda}, u\right)}{\left(z-a_{\lambda}\right)\left(u-b_{\lambda}\right)}+T_{n-1}(z, u) & =\sum_{\nu=0}^{n-1} z^{-\nu} p_{\nu}(z, u)+\bar{P}(z, u) \\
& =\sum_{\nu=0}^{n-2} z^{-\nu} p_{\nu}(z, u)+P(z, u),
\end{aligned}
$$

$$
p_{\nu}(z, u)=\sum_{s=1}^{n} c_{n-1-\nu, n-8} z^{s-1} u^{n-8},
$$

is a homogeneous polynomial in $(z, u)$ of degree $n-1$.

The expression under the sign of integration in (39) we can evidently also reduce to a form like that on the right of (47). In this form further we may here omit terms of degree $n-1$ since in the expression under the sign of integration terms of order higher than $n-2$ do not present themselves.

We may then write

$$
\begin{aligned}
\sum_{e} \frac{\beta_{e} F\left(a_{e}, u\right)}{\left(z-a_{e}\right)\left(u-b_{e}\right)}+T_{n-2}(z, u)=z^{-1} q_{1}(z, u) & +z^{-2} q_{2}(z, u) \\
& +\cdots+z^{-(n-1)} q_{n-1}(z, u)+Q(z, u),
\end{aligned}
$$

where $q_{1}(z, u), \ldots, q_{n-1}(z, u)$ are homogeneous polynomials in $z, u$ of degree $n-1$ and where $Q(z, u)$ is a rational function of $z, u$ which becomes 0 for $z=\infty$.

From (47) and (48) the expression (39) may be written in the form :

$$
\begin{aligned}
\int\left\{z^{-1} q_{1}(z, u)+z^{-2} q_{2}(z, u)+\cdots+z^{-(n-1)} q_{n-1}(z, u)+Q(z, u)\right\} d z \\
-\left\{p_{0}(z, u)+z^{-1} p_{1}(z, u)+\cdots+z^{-(n-2)} p_{n-2}(z, u)+P(z, u)\right\}
\end{aligned}
$$

Supposing now that the expression under the sign of integration is given while the subtracted expression is as yet undetermined, we shall attempt to determine the coefficients $c$ of the polynomials $p_{0}(z, u), p_{1}(z, u), \cdots, p_{n-2}(z, u)$ in such way that the expression in (49) may be finite at $\infty$. 


\section{$\S 9$.}

We have seen in $\S 6$ that a function $z^{r} t(z, u)$, where $t(z, u)$ is a homogeneous polynomial in $z, u$ of degree $n-1$, becomes infinite to the order $n+r-1$ but to no higher order, for one or more of the branches at $\infty$. We had there assumed $r>0$ but the same evidently holds also for negative values of $r$.

The only portion of the expression (49) which might possibly involve infinities at $\infty$ of order $n-1$ is then evidently

$$
\int z^{-1} q_{1}(z, u) d z-p_{0}(z, u) \text {. }
$$

We can however so choose $p_{0}(z, u)$ that the expression just written does not become infinite to an order higher than $n-2$. For, on taking

we shall have:

$$
p_{0}(z, u)=\frac{q_{1}(z, u)}{n-1}
$$

$$
\int z^{-1} q_{1}(z, u) d z-p_{0}(z, u)=\int\left\{z^{-1} q_{1}(z, u)-\frac{1}{n-1} \frac{d}{d z} q_{1}(z, u)\right\} d z
$$

and the expression under the integral sign to the right evidently does not become infinite to the order $n-2$ for any of the branches at $\infty$. On substituting for $d u / d z$ its expression given in (5) we obtain the function

$$
\frac{1}{n-1} \frac{d}{d z} q_{1}(z, u)=\frac{1}{n-1}\left\{\frac{\partial q_{1}}{\partial z}+\frac{\partial q_{1}}{\partial u} \frac{d u}{d z}\right\}
$$

as a rational function of $z, u$ which does not become infinite at $\infty$ to an order higher than $n-2$ nor elsewhere to an order as high as 1 . It is then a function of the type represented in (48) and may therefore be similarly represented. The total expression under the sign of integration to the right of (51) may then also be represented in that form which appears to the right of (48) and we may consequently write :

$$
\begin{aligned}
z^{-1} q_{1}(z, u)-\frac{1}{n-1} \frac{d}{d z} q_{1}(z, u)=z^{-1} q_{1}^{\prime}(z, u)+ & z^{-2} q_{2}^{\prime}(z, u)+\cdots \\
& +z^{-(n-1)} q_{n-1}^{\prime}(z, u)+Q^{\prime}(z, u),
\end{aligned}
$$

where $q_{1}^{\prime}(z, u), \cdots, q_{n-1}^{\prime}(z, u)$ are homogeneous polynomials in $z, u$ of degree $n-1$ and where $Q^{\prime}(z, u)$ is a rational function of $z, u$ which becomes 0 for $z=\infty$. Further, since the expression here represented does not become infinite to the order $n-2$ for any of the branches at $\infty$, we must have identically

$$
q_{1}^{\prime}(z, u)=0 \text {. }
$$


From (51) we shall then have:

$$
\begin{aligned}
\int z^{-1} q_{1}(z, u) d z-p_{0}(z, u)=\int\left\{z^{-2} q_{2}^{\prime}(z, u)\right. & +\cdots \\
& \left.+z^{-(n-1)} q_{n-1}^{\prime}(z, u)+Q^{\prime}(z, u)\right\} d z,
\end{aligned}
$$

and with the aid of this formula we may write our expression in (49) in the form :

where

$$
\begin{aligned}
& \int\left\{z^{-2} q_{2}^{\prime \prime}(z, u)+\cdots+z^{-(n-1)} q_{n-1}^{\prime \prime}(z, u)+Q^{\prime \prime}(z, u)\right\} d z \\
& \quad-\left\{z^{-1} p_{1}(z, u)+z^{-2} p_{2}(z, u)+\cdots+z^{-(n-2)} p_{n-2}(z, u)+P(z, u)\right\},
\end{aligned}
$$

$$
\begin{aligned}
& q_{2}^{\prime \prime}(z, u)=q_{2}(z, u)+q_{2}^{\prime}(z, u), \\
& q_{n-1}^{\prime \prime}(z, u)=q_{n-1}(z, u)+q_{n-1}^{\prime}(z, u) ; \\
& Q^{\prime \prime}(z, u)=Q(z, u)+Q^{\prime}(z, u) \text {. }
\end{aligned}
$$

The expression (53) evidently will not become infinite to an order greater than $n-2$ for any of the branches at $\infty$ and we may further so choose $p_{1}(z, u)$ that it will not become infinite even to this order.

For the only portion of the expression (53) which might possibly involve infinities at $\infty$ of order $n-2$ is evidently

and on choosing

$$
\int z^{-2} q_{2}^{\prime \prime}(z, u) d z-z^{-1} p_{1}(z, u),
$$

we shall have:

$$
p_{1}(z, u)=\frac{q_{2}^{\prime \prime}(z, u)}{n-2}
$$

$$
\int z^{-2} q_{2}^{\prime \prime}(z, u) d z-z^{-1} p_{1}(z, u)=\int\left\{z^{-2} q_{2}^{\prime \prime}(z, u)-\frac{d}{d z} \frac{z^{-1} q_{2}^{\prime \prime}(z, u)}{n-2}\right\} d z
$$

where, on substituting for $u$ in terms of $z$ from the equation to a branch at $\infty$, the expression under the sign of integration on the right will evidently not be infinite to an order higher than $n-4$ and the integral itself therefore to an order not higher than $n-3$.

This expression we may write in the form:

$$
z^{-2} q_{2}^{\prime \prime}(z, u)-\frac{1}{n-2} \frac{d}{d z} z^{-1} q_{2}^{\prime \prime}(z, u)=\frac{n-1}{n-2} z^{-2} q_{2}^{\prime \prime}(z, u)-\frac{z^{-1}}{n-2} \frac{d q_{2}^{\prime \prime}(z, u)}{d z} .
$$

As in the case of $d q_{1}(z, u) / d z$ so here $d q_{2}^{\prime \prime}(z, u)_{/}^{\prime} d z$ - and in fact the derivative of any homogeneous polynomial in $z, u$ of degree $n-1$-is of the type of function represented in (48), and the expression just written may therefore evidently be written in the form : 


$$
\begin{aligned}
\frac{n-1}{n-2} z^{-2} q_{2}^{\prime \prime}(z, u)-\frac{z^{-1}}{n-2} \frac{d q_{2}^{\prime \prime}(z, u)}{d z}=z^{-2} q_{2}^{\prime \prime \prime}(z, u) & +z^{-3} q_{3}^{\prime \prime \prime}(z, u) \cdots \\
& +z^{-(n-1)} q_{n-1}^{\prime \prime \prime}(z, u)+Q^{\prime \prime \prime}(z, u),
\end{aligned}
$$

where $q_{2}^{\prime \prime \prime}(z, u), \cdots, q_{n-1}^{\prime \prime \prime}(z, u)$ are homogeneous polynomials in $z, u$ of degree $n-1$ and where $Q^{\prime \prime \prime}(z, u)$ is a rational function of $z, u$ which becomes 0 for $z=\infty$. Further, since the expression here represented does not become infinite to the order $n-3$ for any of the branches at $\infty$, we must have identically

$$
q_{2}^{\prime \prime \prime}(z, u)=0 \text {. }
$$

From (54) we shall then have :

$$
\begin{aligned}
\int z^{-2} q_{2}^{\prime \prime}(z, u) d z-z^{-1} p_{1}(z, u)=\int\left\{z^{-3} q_{3}^{\prime \prime \prime}(z, u)+z^{-4} q_{4}^{\prime \prime \prime}(z, u)+\cdots\right. \\
\\
\left.+z^{-(n-1)} q_{n-1}^{\prime \prime \prime}(z, u)+Q^{\prime \prime \prime}(z, u)\right\} d z,
\end{aligned}
$$

and with the aid of this formula we may write the expression in (53) and therefore that in (49) in the form

$$
\begin{array}{r}
\int\left\{z^{-3} q_{3}^{(4)}(z, u)+\cdots+z^{-(n-1)} q_{n-1}^{(4)}(z, u)+Q^{(4)}(z, u)\right\} d z-\left\{z^{-2} p_{2}(z, u)\right. \\
\left.+\cdots+z^{-(n-2)} p_{n-2}(z, u)+P(z, u)\right\},
\end{array}
$$

where

$$
\begin{aligned}
& q_{3}^{(4)}(z, u)=q_{3}^{\prime \prime}(z, u)+q_{3}^{\prime \prime \prime}(z, u), \\
& q_{n-1}^{(4)}(z, u)=q_{n-1}^{\prime \prime}(z, u)+q_{n-1}^{\prime \prime \prime}(z, u) ; \\
& Q^{(4)}(z, u)=Q^{\prime \prime}(z, u)+Q^{\prime \prime \prime}(z, u) \text {. }
\end{aligned}
$$

The expression (56) will evidently not become infinite to an order greater than $n-3$ for any of the branches at $\infty$ and we might so choose $p_{2}(z, u)$ that it would not become infinite even to this order. Suppose now that by proper choice of the polynomials $p_{2}(z, u), \cdots, p_{r-1}(z, u)$ we have reduced (56) and therewith (49) to the form

$$
\begin{aligned}
\int\left\{z^{-(r+1)} q_{r+1}^{(2 r)}(z, u)+\right. & \left.\cdots+z^{-(n-1)} q_{n-1}^{(2 r)}(z, u)+Q^{(2 r)}(z, u)\right\} d z \\
& -\left\{z^{-r} p_{r}(z, u)+\cdots+z^{-(n-2)} p_{n-2}(z, u)\right\}+P(z, u),
\end{aligned}
$$

where $q_{r+1}^{(2 r)}(z, u), \cdots, q_{n-1}^{(2 r)}(z, u)$ are homogeneous polynomials in $z, u$ of degree $n-1$ and where $Q^{(2 r)}(z, u)$ is a rational function of $z, u$ which becomes 0 for $z=\infty$. This expression will evidently not become infinite to an order greater than $n-r-1$ for any of the branches at $\infty$ and we may so choose $p_{r}(z, u)$ that it will not become infinite to an order greater than $n-r-2$. 
For the only portion of the expression (57) which might possibly involve infinities at $\infty$ of order $n-r-1$ is evidently

and on choosing

$$
\int z^{-(r+1)} q_{r+1}^{(2 r)}(z, u) d z-z^{-r} p_{r}(z, u),
$$

we shall have :

$$
p_{r}(z, u)=\frac{q_{r+1}^{(2 r)}(z, u)}{n-r-1}
$$

$$
\begin{aligned}
& \int z^{-(r+1)} q_{r+1}^{(2 r)}(z, u) d z-z^{-r} p_{r}(z, u) \\
&= \int\left\{z^{-(r+1)} q_{r+1}^{(2 r)}(z, u)-\frac{d z^{-r} q_{r+1}^{(2 r)}(z, u)}{d z}\right\} d z, \\
& n-r-1
\end{aligned}
$$

where, on substituting for $u$ in terms of $z$ from the equation to a branch at $\infty$, the expression under the sign of integration on the right will evidently not be infinite to an order higher than $n-r-3$ and the integral itself therefore to an order not higher than $n-r-2$.

This expression we may write in the form :

$$
\begin{aligned}
& z^{-(r+1)} q_{r+1}^{(2 r)}(z, u)-\frac{1}{n-r-1} \frac{d}{d z} z^{-r} q_{r+1}^{(2 r)}(z, u) \\
& \quad=\frac{n-1}{n-r-1} z^{-(r+1)} q_{r+1}^{(2 r)}(z, u)-\frac{z^{-r}}{n-r-1} \frac{d q_{r+1}^{(2 r)}(z, u)}{d z} .
\end{aligned}
$$

The derivative $d q_{r+1}^{(2 r)}(z, u) /(d z)$ is of the type of function represented in (48) and the expression with which we here have to do may evidently therefore be represented in the form :

$$
\begin{array}{r}
\frac{n-1}{n-r-1} z^{-(r+1)} q_{r+1}^{(2 r)}(z, u)-\frac{z^{-r}}{n-r-1} \frac{d q_{r+1}^{(2 r)}(z, u)}{d z}=z^{-(r+1)} q_{r+1}^{(2 r+1)}(z, u) \\
+z^{-(r+2)} q_{r+2}^{(2 r+1)}(z, u)+\cdots+z^{-(n-1)} q_{n-1}^{(2 r+1)}(z, u)+Q^{(2 r+1)}(z, u),
\end{array}
$$

where $q_{r+1}^{(2 r+1)}(z, u), \cdots, q_{n-1}^{(2 r+1)}(z, u)$ are homogeneous polynomials in $z, u$ of degree $n-1$ and where $Q^{(2 r+1)}(z, u)$ is a rational function of $z, u$ which becomes 0 for $z=\infty$. Further since, as we have just seen, the expression here represented does not become infinite to an order higher than $n-r-3$ for any of the branches at $\infty$, we must have identically

$$
q_{r+1}^{(2 r+1)}(z, u)=0 .
$$

From (58) we shall then have :

$$
\begin{aligned}
& \int z^{-(r+1)} q_{r+1}^{(2 r)}(z, u) d z- z^{-r} p_{r}(z, u)=\int\left\{z^{-(r+2)} q_{r+2}^{(2 r+1)}(z, u)\right. \\
&\left.+\cdots+z^{-(n-1)} q_{n-1}^{(2 r+1)}(z, u)+Q^{(2 r+1)}(z, u)\right\} d z
\end{aligned}
$$


and with the aid of this formula we may write the expression in (57) and therewith that in (49) in the form

$$
\begin{aligned}
\int\left\{z^{-(r+2)} q_{r+2}^{(2 r+2)}(z, u)+\cdots+z^{-(n-1)} q_{n-1}^{(2 r+2)}(z, u)+Q^{(2 r+2)}(z, u)\right\} d z \\
-\left\{z^{-(r+1)} p_{r+1}(z, u)+\cdots+z^{-(n-2)} p_{n-2}(z, u)+P(z, u)\right\}
\end{aligned}
$$

where

$$
\begin{gathered}
q_{r+2}^{(2 r+2)}(z, u)=q_{r+2}^{(2 r)}(z, u)+q_{r+2}^{(2 r+1)}(z, u) \\
\cdot \cdot \cdot \cdot \\
q_{n-1}^{(2 r+2)}(z, u)=q_{n-1}^{(2 r)}(z, u)+q_{n-1}^{(2 r+1)}(z, u) \\
Q^{(2 r+2)}(z, u)=Q^{(2 r)}(z, u)+Q^{(2 r+1)}(z, u) .
\end{gathered}
$$

By induction then we see that a proper choice of the homogeneous polynomials $p_{0}(z, u), p_{1}(z, u), \cdots, p_{\dot{n}_{-2}}(z, u)$ will enable us to reduce $(49)$ to the form

$$
\int Q^{(2 n-2)}(z, u) d z-P(z, u)
$$

where $Q^{(2 n-2)}(z, u)$ is a rational function of $(z, u)$ which becomes equal to 0 for $z=\infty$. Since also $P(z, u)$ is finite for $z=\infty$ it follows that the expression (61) cannot become algebraically infinite at $\infty$. That it cannot become logarithmically infinite is also evident, for the integral in the equivalent expression (49), being only another form for the integral in (39), does not become infinite loyarithmically.

We see then that the $n(n-1)$ coefficients in the $n-1$ polynomials $p_{0}(z, u)$, $p_{1}(z, u), \cdots, p_{n-2}(z, u)$ may be so chosen that the expression in (49) does not become infinite for $z=\infty$, and further we may show that there is only one way of so choosing them. For if we had two systems of functions $p_{0}(z, u)$, $p_{1}(z, u), \cdots, p_{n-2}(z, u), P(z, u)$ and $p_{0}^{\prime}(z, u), p_{1}^{\prime}(z, u), \cdots, p_{n-2}^{\prime}(z, u), P^{\prime}(z, u)$ corresponding to two determinations of these coefficients, the expression

$$
\begin{aligned}
\int\left\{z^{-1} q_{1}(z, u)\right. & \left.+z^{-2} q_{2}(z, u)+\cdots+z^{-(n-1)} q_{n-1}(z, u)+Q(z, u)\right\} d z \\
& -\left\{p_{0}^{\prime}(z, u)+z^{-1} p_{1}^{\prime}(z, u)+\cdots+z^{-(n-2)} p_{n-2}^{\prime}(z, u)+P^{\prime}(z, u)\right\},
\end{aligned}
$$

and that in (49) would both be finite for $z=\infty$. The difference between these two expressions would then be finite for $z=\infty$ and, since the functions $P(z, u)$ and $P^{\prime}(z, u)$ are finite for this value of $z$, this also would be the case for the difference,

$$
\begin{aligned}
\left\{p_{0}(z, u)+z^{-1} p_{1}(z, u)+\right. & \left.\cdots+z^{-(n-2)} p_{n-2}(z, u)\right\} \\
& -\left\{p_{0}^{\prime}(z, u)+z^{-1} p_{1}^{\prime}(z, u)+\cdots+z^{-(n-2)} p_{n-2}^{\prime}(z, u)\right\} .
\end{aligned}
$$

From this however evidently follows that we must have identically:

$$
p_{0}(z, u)=p_{0}^{\prime}(z, u), \quad p_{1}(z, u)=p_{1}^{\prime}(z, u), \cdots, p_{n-2}(z, u)=p_{n-2}^{\prime}(z, u) .
$$


We see then that there is one and only one determination of the coefficients in the $n-1$ polynomials $p_{0}(z, u), p_{1}(z, u), \cdots, p_{n-2}(z, u)$, such that the expression in (49) does not become infinite for $z=\infty$.

\section{$\S 10$.}

The $n(n-1)$ coefficients of the polynomials $p_{0}(z, u), \cdots, p_{n-2}(z, u)$ in $(47)$ are those which have been indicated by

$$
\begin{aligned}
& c_{n-1, n-1}, \quad c_{n-1, n-2}, \cdots, c_{n-1,0}, \\
& c_{n-2, n-1}, \quad c_{n-2, n-2}, \cdots, c_{n-2,0} \text {, } \\
& c_{n-3, n-1}, \quad c_{n-3, n-2}, \cdots, c_{n-3,0} \text {, } \\
& c_{1, n-1} \quad, c_{1, n-2}, \cdots, c_{1,0} \text {. }
\end{aligned}
$$

To these coefficients we shall suppose to be assigned that system of values for which the expression (49) does not become infinite at $\infty$.

With those coefficients in which the first suffix is equal to or greater than the second, we shall construct a polynomial

$$
T_{n-1}(z, u)=\sum_{s=1}^{n} \sum_{r=0}^{s-1} c_{n-s+r, n-s} z^{r} u^{n-s},
$$

in which the constant term $c_{00}$ may have any arbitrary value.

Corresponding to the $\frac{1}{2}(n-1)(n-2)$ coefficients

$$
\begin{aligned}
& c_{n-2, n-1} ; \\
& c_{n-3, n-1}, c_{n-3, n-2} ; \\
& \cdot \cdot \cdot \cdot . \cdot \cdot \\
& c_{1, n-1}, c_{1, n-2}, \ldots, c_{1,2},
\end{aligned}
$$

in which the first suffix is smaller than the second, we shall have as many equations of the type given in (44). They will be obtained on giving to $r$ and $s$ respectively in (44) the values

$$
r=1,2, \ldots, n-s-1 ; s=1,2, \ldots, n-2 .
$$

This system of $\frac{1}{2}(n-1)(n-2)$ equations we shall attempt to satisfy by a proper choice of the quantities $\gamma_{i \kappa}$ on the right. The number of these quantities which present themselves in the $\frac{1}{2}(n-1)(n-2)$ equations is just equal to the number of the equations as we may readily show. For the greatest value for the sum of the suffixes $i$ and $\kappa$ in a term on the right of (44) is equal to $r+s$ -2 , and $r+s-2$, as we see from (64), cannot exceed $n-3$ in value. It follows therefore that the sum of the suffixes in the quantities $\gamma_{i \kappa}$ which ap- 
pear in our equations, is in no case greater than $n-3$. Now the number of possible pairs of integers $i, \kappa$ whose sum is equal to or less that $n-3$ is $\frac{1}{2}(n-1)(n-2)$, and that all the $\frac{1}{2}(n-1)(n-2)$ corresponding quantities $\gamma_{i \kappa}$ actually present themselves in our system of equations is immediately apparent. In fact each one of them appears in some one of the equations (44) with $e_{0 n}$ as coefficient as we see on taking $\rho=0, \sigma=0$ and substituting for $r, s$ in the corresponding term $e_{0, n} \gamma_{r-1,8-1}$ the several pairs of values furnished by (64).

The number of the undetermined quantities $\gamma_{i_{\kappa}}$ which present themselves in the system of equations (44) is then just equal to $\frac{1}{2}(n-1)(n-2)$, the number of these equations.

From the equations (44) we shall now seek to determine the quantities $\gamma_{i_{\kappa}}$ in terms of the quantities $c_{n-s-r, n-s}$. For this purpose we shall write our equations in the form :

$$
\begin{aligned}
c_{n-s-r, n-s}=e_{0, n} \gamma_{r-1, s-1} & +e_{1, n-1} \gamma_{r, s-2}+e_{2, n-2} \gamma_{r+1, s-3}+\cdots \\
& +e_{s-2, n-s+2} \gamma_{r+s-3,1}+e_{s-1, n-s+1} \gamma_{r+s-2,0}+\Gamma_{r-1, s-1},
\end{aligned}
$$

where $\Gamma_{r-1, s-1}$ depends only on quantities $\gamma_{i_{\kappa}}$, the sum of whose suffixes is less than $r+s-2$.

The number of equations of the type (65), in which the sum of the suffixes of the quantities $\gamma$ in evidence on the right has a given value $r+s+2=\sigma$, is $\sigma+1$ and these $\sigma+1$ equations may evidently be written in the form :

$$
\begin{aligned}
& e_{0, n} \gamma_{0, \sigma}+e_{1, n-1} \gamma_{1, \sigma-1}+e_{2, n-2} \gamma_{2, \sigma-2}+\cdots+e_{\sigma-1, n-\sigma+1} \gamma_{\sigma-1,1}+e_{\sigma, n-\sigma} \gamma_{\sigma, 0} \\
& =c_{n-\sigma-2, n-\sigma-1}-\Gamma_{0, \sigma}, \\
& e_{0, n} \gamma_{1, \sigma-1}+e_{1, n-1} \gamma_{2, \sigma-2}+\cdots+e_{\sigma-2, n-\sigma+2} \gamma_{\sigma-1,1}+e_{\sigma-1, n-\sigma+1} \gamma_{\sigma, 0} \\
& =c_{n-\sigma-2, n-\sigma}-\Gamma_{1, \sigma-1} \text {, } \\
& e_{0, n} \quad \gamma_{2, \sigma-2}+\cdots+e_{\sigma-3, n-\sigma+3} \gamma_{\sigma-1,1}+e_{\sigma-2, n-\sigma+2} \gamma_{\sigma, 0} \\
& =c_{n-\sigma-2, n-\sigma+1}-\Gamma_{2, \sigma-2} \text {, } \\
& e_{0, n} \gamma_{\sigma-1,1}+e_{1, n-1} \gamma_{\sigma, 0} \\
& =c_{n-\sigma-2, n-2}-\Gamma_{\sigma-1,1} \text {, } \\
& e_{0, n} \gamma_{\sigma, 0} \\
& =c_{n-\sigma-2, n-1}-\Gamma_{\sigma, 0} \text {. }
\end{aligned}
$$

If now we suppose that the quantities $\gamma_{i_{\kappa}}$ in which the sum of the suffixes is less than $\sigma$-and by consequence also the quantities $\Gamma$ to the right of (66)-have been obtained in terms of the quantities $c$, the system of equations (66) will 
determine in terms of these latter quantities the quantities $\gamma_{i_{\kappa}}$ also, in which the sum of the suffixes is equal to $\sigma$. For the determinant of the coefficients on the left of (66) is different from 0 and has in fact the value $e_{0, n}^{\sigma+1}=1$, since in our original equation $F(z, u)=0$ we assumed $e_{0, n}=1$.

More precisely still, on assuming that the quantities $\gamma_{i_{\kappa}}$, in which the sum of the suffixes is less than $\sigma$, are linearly expressible in terms of the quantities $c$, it follows from the system of equations (66) that the quantities $\gamma_{i \kappa}$ in which the sum of the suffixes is equal to $\sigma$ are also so expressible, and these equations furnish us.with their expressions in terms of the $c$ 's .

Consider now the case $\sigma=0$. In this case the system of equations (66) reduces to the single equation

$$
e_{0, n} \gamma_{0,0}=c_{n-2, n-1},
$$

so that the quantity $\gamma_{00}$, in accord with our hypothesis above, is linearly expressible in terms of the quantities $c$ and in fact is equal to one of them, namely $c_{n-2, n-1}$. It follows therefrom by our inductive reasoning above, that the quantities $\gamma_{01}$ and $\gamma_{10}$ corresponding to the case $\sigma=1$ are also linearly expressible in terms of the quantities $c$, and by successive induction we see that the quantities $\gamma_{i_{\kappa}}$ for which $\sigma(=i+\kappa)$ has one of the values $2,3, \ldots, n-3$, are all linearly expressible in terms of the quantities $c$, the expressions for the same being furnished by the system of equations (44) supposed for that purpose to be grouped in a series of sets of the type given in (66).

The values of the $\frac{1}{2}(n-1)(n-2)$ quantities $\gamma_{i_{\kappa}}$, so obtained, we may suppose to be substituted on the left in the corresponding equations of the type (43), when expressions for the $d+p=\frac{1}{2}(n-1)(n-2)$ quantities $\gamma_{\lambda}$ in terms of our quantities $\gamma_{i_{\kappa}}$ will be furnished by these equations in case we have

$$
\left|a_{\lambda}^{i} b_{\lambda}^{\kappa}\right| \neq 0 \quad(i+\kappa=0,1,2, \cdots, n-3),
$$

where by $\left|a_{\lambda}^{i} b_{\lambda}^{k}\right|$ we indicate the determinant of the coefficients of the quantities $\gamma_{\lambda}$ to the right of our equations (43). That the determination so made of the quantities $\gamma_{\lambda}$ is unique is self-evident.

In the case where we have $\left|a_{\lambda}^{i} b_{\lambda}^{\kappa}\right|=0$ it will be impossible to satisfy the system of equations (43) by any set of values $\gamma_{\lambda}$.unless it happens that a certain relation exists between the quantities $\gamma_{i_{\kappa}}$. Such a relation however does not in general exist, as we shall show later on.

Assuming then that our system of points $\left(a_{\lambda}, b_{\lambda}\right)$ has been so chosen that the determinant above has a value other than 0 , or, what evidently amounts to the same thing, in such way that the points do not all lie on a curve of degree $n+3$, we shall suppose that the function

$$
\sum_{\lambda=1}^{a+p} \frac{\gamma_{\lambda} F\left(a_{\lambda}, u\right)}{\left(z-a_{\lambda}\right)\left(u-b_{\lambda}\right)}+T_{n-1}(z, u)
$$


in (39) has been constructed with the values of the $d+p$ quantities $\gamma_{\lambda}$ furnished by the equations (43) and with the polynomial $T_{n-1}(z, u)$ obtained in (62). Apart from the arbitrary constant in $T_{n-1}(z, u)$ the expression (39) so determined is unique.

We have shown that if the expression (39) is to be finite for all the branches at $\infty$ it must be determined as just stated. Conversely in case it has the determination here in question it will be finite for $z=\infty$.

For the coefficients $\gamma_{\lambda}$ in (39) have been derived through the equations (43) and (44) from certain of the coefficients $c$ in the polynomials $p_{0}(z, u), p_{1}(z, u)$, $\cdots, p_{n-2}(z, u)$, these polynomials having been at the same time assumed by us to be those for which the expression (49) is finite at $\infty$; and conversely, from the values thus obtained for the coefficients $\gamma_{\lambda}$ through the equations (43) and (44) in the reverse order, we should evidently arrive at the original coefficients $c$ in question. Also the remaining coefficients $c$ in the polynomials $p_{0}(z, u)$, $p_{1}(z, u), \ldots, p_{n-2}(z, u)$ are identical with those in $T_{n-1}(z, u)$-the constant term $c_{00}$ in the last named function being of course left out of consideration-so that the polynomials $p_{0}(z, u), \cdots, p_{n-2}(z, u)$ in (49), regarded as a reduced form of the expression (39), are those for" which the expression (49) was originally assumed to be finite at $\infty$. It follows therefore that the uniquely determined expression (39) is finite for $z=\infty$.

To recapitulate-we have proved in the above that in the expression (39) the coefficients $\gamma_{\lambda}$ and the coefficients in the polynomial $T_{n-1}(z, u)$ can be chosen, and that uniquely, in such way that this expression does not become infinite for $z=\infty$ in case we have so selected our $d+p$ points $\left(a_{\lambda}, b_{\lambda}\right)$ that they do not lie on a curve of degree $n-3$, and we have furnished a method for determining the coefficients in question.

We have assumed from the outset that the $d$ double points are included under the $d+p$ points $\left(a_{\lambda}, b_{\lambda}\right)$ in the expression (39). It might however be remarked in this connection that all our reasoning above in regard to this expression holds good for any $d+p$ arbitrary points. That we can choose $d+p$ points $\left(a_{\lambda}, b_{\lambda}\right)$ which do not lie on a curve of degree $n-3$ is self-evident, for $d+p=\frac{1}{2}(n-1)(n-2)$ is the number of coefficients in the general equation to a curve of degree $n-3$.

That however, among $d+p$ points so chosen we may include the $d$ double points is not self-evident. It may however be proved that the passage through the double points imposes $d$ conditions on our curve, or in other words that the conditions of adjointness are independent of one another.

I shall not stop here to prove the theorem in question but merely remark that the methods of the present paper furnish an extremely simple algebraic proof of it, which I propose to give in another paper at an early date. Meantime I shall accept the principle of the independence of the conditions of adjointness as Trans. Am. Math. Soc. 6 
demonstrated, and we may therefore assume that the $d$ double points are included among the $d+p$ points $\left(a_{\lambda}, b_{\lambda}\right)$; and in (39), whatever the values of the constants in the expression under the sign of integration, we may regard the coefficients $\gamma_{\lambda}$ and the coefficients in the polynomial $T_{n-1}(z, u)$ in the subtracted function as so determined that the difference does not become infinite for $z=\infty$.

$\S 11$.

Instead of (39) I shall now consider the expression

$$
\begin{aligned}
\int\left\{\sum_{e} \frac{\beta_{e} F\left(a_{e}, u\right)}{\left(z-a_{e}\right)\left(u-b_{e}\right)}+T_{n-2}(z, u)\right\} d z \\
-\left\{\sum_{\lambda=1}^{d+p}\left(\frac{\gamma_{\lambda} F\left(a_{\lambda}, u\right)}{\left(z-a_{\lambda}\right)\left(u-b_{\lambda}\right)}+m_{\lambda} \mathrm{II}\left(a_{\lambda}, b_{\lambda}\right)\right)+T_{n-1}(z, u)\right\},
\end{aligned}
$$

where by $\operatorname{II}\left(a_{\lambda}, b_{\lambda}\right)$ we indicate an elementary integral of the second kind possessing a single infinity of the first order at the point $\left(a_{\lambda}, b_{\lambda}\right)$, and where the coefficients $m_{\lambda}$ may be supposed to be so determined that the expressions

$$
\frac{\gamma_{\lambda} F\left(a_{\lambda}, u\right)}{\left(z-a_{\lambda}\right)\left(u-\overline{b_{\lambda}}\right)}+m_{\lambda} \mathrm{II}\left(a_{\lambda}, b_{\lambda}\right)
$$

do not become infinite at the points $\left(a_{\lambda}, b_{\lambda}\right)$.

The residues of the integrals $\operatorname{II}\left(a_{\lambda}, b_{\lambda}\right)$ we may assume to have the value 1 , and the coefficients $m_{\lambda}$ will then evidently be determined by the equations

$$
\gamma_{\lambda} F_{b_{\lambda}}^{\prime}\left(a_{\lambda}, b_{\lambda}\right)+m_{\lambda}=0 \text {. }
$$

In the case of the double points the coefficients $m_{\lambda}$ will of course have the value 0 .

The expression (68) does not possess infinities other than those of (39) and it does not possess even these, for the infinities of the latter expression are all to be found among the points $\left(a_{\lambda}, b_{\lambda}\right)$, for all of which (68) is finite. The expression (68) is then everywhere finite and must therefore be an integral of the first kind. On indicating this integral by $I$ and on supposing $\left(a_{p+1}, b_{p+1}\right),\left(a_{p+2}, b_{p+2}\right)$, $\cdots,\left(a_{p+d}, b_{p+d}\right)$ to denote the $d$ double points we may write $(68)$ in the form :

$$
\begin{aligned}
\int\left\{\sum_{e} \frac{\beta_{e} F\left(a_{e}, u\right)}{\left(z-a_{e}\right)\left(u-b_{e}\right)}+T_{n-2}(z, u)\right\} d z-\left\{\sum_{\lambda=1}^{d+p} \frac{\gamma_{\lambda} F\left(a_{\lambda}, u\right)}{\left(z-a_{\lambda}\right)\left(u-b_{\lambda}\right)}\right. & \\
+ & \left.T_{n-1}(z, u)\right\}-\sum_{\lambda=1}^{p} m_{\lambda} \mathrm{II}\left(a_{\lambda}, b_{\lambda}\right)=\mathrm{I} .
\end{aligned}
$$

Returning now to formula (35) and substituting therein for the integral

$$
\int\left\{\sum_{e} \frac{\beta_{e} F\left(a_{e}, u\right)}{\left(z-a_{e}\right)\left(u-b_{e}\right)}+T_{n-2}(z, u)\right\} d z
$$


the expression derived from (69), we obtain :

$$
\begin{aligned}
\int R(z, u) d z=\bar{G}(z, u)+\sum_{\lambda=1}^{d+p} \frac{\gamma_{\lambda} F\left(a_{\lambda}, u\right)}{\left(z-a_{\lambda}\right)\left(u-b_{\lambda}\right)}+T_{n-1}(z, u)+\mathrm{I} & \\
& +\sum_{\lambda=1}^{p} m_{\lambda} \mathrm{II}\left(a_{\lambda}, b_{\lambda}\right)+\mathrm{III} .
\end{aligned}
$$

Indicating the aggregate rational portion of this expression by $G(z, u)$ we have:

$$
\int R(z, u) d z=G(z, u)+\mathrm{I}+\sum_{\lambda=1}^{p} m_{\lambda} \mathrm{II}\left(a_{\lambda}, b_{\lambda}\right)+\mathrm{III} .
$$

Our original Abelian integral $\int R(z, u) d z$ is herewith reduced to the sum of a rational function, an integral of the first kind, a linear function of $p$ elementary integrals of the second kind and an integral of the third kind, the several steps in the process thereto employed being all of algebraic character. From a glance at the process also, it is plain that the coefficients $m_{\lambda}$ and the function $G(z, u)$, apart from the arbitrary constant of integration, are completely determined when the system of points $\left(a_{1}, b_{1}\right), \cdots,\left(a_{p}, b_{p}\right)$ is given.

\section{$\S 12$.}

We have effected the reduction of the general Abelian integral indicated in (70), on the hypothesis that the $p$ points $\left(a_{1}, b_{1}\right), \ldots,\left(a_{p}, b_{p}\right)$ do not lie on an adjoint curve of degree $n-3$. In the case where they do lie on an adjoint curve of degree $n-3$ it may readily be shown that such a reduction is not in general possible.

For suppose that we have a system of $p$ points

$$
\left(\bar{a}_{1}, \bar{b}_{1}\right), \cdots,\left(\bar{a}_{p}, \bar{b}_{p}\right)
$$

lying on an adjoint curve of degree $n-3$ and that the $p$ points

$$
\left(a_{1}, b_{1}\right), \cdots,\left(a_{p}, b_{p}\right)
$$

do not all lie on such a curve.

Referring now to the expression in (39), which we sought to render finite at $\infty$ by proper choice of the coefficients $\gamma_{\lambda}$ and of the polynomial $T_{n-1}(z, u)$ and regarding in particular the formulæ in (43), (44), (49), we remark that the constants $c$ are independent of our choice of the $p$ points $\left(a_{1}, b_{1}\right), \ldots,\left(a_{p}, b_{p}\right)$. From the form in (49), namely, we see that they depend for their values on the integral

$$
\int\left\{z^{-1} q_{1}(z, u)+z^{-2} q_{2}(z, u)+\cdots+z^{-(n-1)} q_{n-1}(z, u)+Q(z, u)\right\} d z
$$


alone, or what is the same thing, on the integral

$$
\int\left\{\sum_{e} \frac{\beta_{e} F\left(a_{e}, u\right)}{\left(z-a_{e}\right)\left(u-b_{c}\right)}+T_{n-2}(z, u)\right\} d z
$$

in (39), independently of what our choice of the system of $p$ points $\left(a_{1}, b_{1}\right)$, $\cdots ;\left(a_{p}, b_{p}\right)$ may happen to be.

From the equations (44) again the quantities $\gamma_{i \kappa}$ depend upon the quantities $c$ alone and are therefore also independent of the choice of our system of points $\left(a_{1}, b_{1}\right), \cdots,\left(a_{p}, b_{p}\right)$.

In our system of equations (43), then, the quantities $\gamma_{i \kappa}$ on the left have the same values whatever the $p$ points $\left(a_{1}, b_{1}\right), \ldots,\left(a_{p}, b_{p}\right)$ may be and depend solely on the integral in (39).

To prove that we cannot employ the system of $p$ points $\left(\bar{a}_{1}, \bar{b}_{1}\right), \ldots,\left(\bar{a}_{p}, \bar{b}_{p}\right)$ for the reduction of the general Abelian integral it will suffice to show in some particular case that the quantities $\gamma_{i \kappa}$ corresponding to an integral of the type of that in (39) are such that the system of equations

$$
\gamma_{i \kappa}={ }_{\lambda=1}^{d+p} \bar{\gamma}_{\lambda} \bar{a}_{\lambda}^{i} \bar{b}_{\lambda}^{\kappa}
$$

cannot be satisfied. The $d$ double points for convenience I shall designate indifferently by $\left(a_{p+1}, b_{p+1}\right), \ldots,\left(a_{p+d}, b_{p+d}\right)$ or $\left.\left(\bar{a}_{p+1}, \bar{b}_{p+1}\right), \ldots, \bar{a}_{p+d}, \bar{b}_{p+d}\right)$. Consider now in particular the function

$$
\sum_{\lambda=1}^{p+d} \frac{\gamma_{\lambda} F\left(a_{\lambda}, u\right)}{\left(z-a_{\lambda}\right)\left(u-b_{\lambda}\right)}+\sum_{\lambda=1}^{p} \eta_{\lambda} \operatorname{II}\left(a_{\lambda}, b_{\lambda}\right),
$$

where we suppose the coefficients $m_{\lambda}$ to be so determined that the function does not become infinite for any of the points $\left(a_{\lambda}, b_{\lambda}\right)$. The derivative of this function with regard to $z$ will not become infinite to an order as high as 1 for any finite point, and for the points at $\infty$ it will evidently not become infinite to an order higher than $n-3$ or, what is sufficient for our purpose, it will not become infinite at $\infty$ to an order higher than $n-2$. This derivative therefore will plainly be of that type of function which appears under the sign of integration in (39).

On properly determining the coefficients $\beta$ and the polynomial $T_{n-2}(z, u)$, then, we may write :

$$
\begin{gathered}
\sum_{e} \frac{\beta_{e} F\left(a_{e}, u\right)}{\left(z-a_{e}\right)\left(u-b_{e}\right)}+T_{n-2}(z, u)=\frac{d}{d z}\left\{\sum_{\lambda=1}^{p+d} \frac{\gamma_{\lambda} F\left(a_{\lambda}, u\right)}{\left(z-a_{\lambda}\right)\left(u-b_{\lambda}\right)}\right. \\
\left.\quad+\sum_{\lambda=1}^{p} m_{\lambda} \operatorname{II}\left(a_{\lambda}, b_{\lambda}\right)\right\} .
\end{gathered}
$$


The difference

$$
\int\left\{\sum_{e} \frac{\beta_{e} F\left(a_{e}, u\right)}{\left(z-a_{e}\right)\left(u-b_{\odot}\right)}+T_{n-2}(z, u)\right\} d z+\sum_{\lambda=1}^{d+p} \frac{\gamma_{\lambda} F\left(a_{\lambda}, u\right)}{\left(z-a_{\lambda}\right)\left(u-b_{\lambda}\right)}
$$

is then evidently an expression of the type (39) which does not become infinite for $z=\infty$.

Here the quantities $\gamma_{i_{\kappa}}$ corresponding to the integral in (72) are given by the system of equations :

$$
\gamma_{i \kappa}=\sum_{\lambda=1}^{d+p} \gamma_{\lambda} a_{\lambda}^{i} b_{\lambda}^{\kappa}
$$

and on starting out from a properly chosen set of values for the quantities $\gamma_{\lambda}$ we could arrive at any arbitrarily assigned system of values for the quantities $\gamma_{i_{\kappa}}$, since for the determinant of the coefficients of the quantities $\gamma_{\lambda}$ to the right of these equations we have $\left|a_{\lambda}^{i} b_{\lambda}^{k}\right| \neq 0$. To this assigned system of values for the quantities $\gamma_{i \kappa}$ will correspond a certain set of values $\gamma_{\lambda}$ and therewith a certain integral of the type in (72).

Now for the reduction of this integral the system of $p$ points $\left(\bar{a}_{1}, \bar{b}_{1}\right), \ldots$, $\left(\bar{a}_{p}, \bar{b}_{p}\right)$ will not serve if values have been assigned to the quantities $\gamma_{i_{\kappa}}$ for which the equations (71) cannot be solved. Such values, however, it is always possible to assign to these quantities since for the determinant of the coefficients of the quantities $\bar{\gamma}$ to the right of these equations we have $\left|\bar{a}_{\lambda}^{i}, \bar{b}_{\lambda}^{\kappa}\right|=0$. It follows then that a system of $p$ points $\left(\bar{a}_{1}, \bar{b}_{1}\right), \ldots,\left(\bar{a}_{p}, \bar{b}_{p}\right)$ which lie on an adjoint curve of degree $n-3$ cannot serve for the reduction of the general Abelian integral.

It will be remembered that in obtaining the reduced form on the right of (70), we assumed that no one of the $p$ points $\left(a_{1}, b_{1}\right), \cdots,\left(a_{p}, b_{p}\right)$ was a double point. The only limitation otherwise placed upon our choice of these points was that they should not all be zeros of one and the same adjoint curve of degree $(n-3)$.

It may however be remarked that in obtaining the reduced form in question, we might also have included under the $p$ elementary integrals of the second kind integrals corresponding to the double points, or more accurately speaking, to certain of the branches through the double points.

To have done this it would have been necessary to introduce some modifica tions into our reasoning which would have added to the length of the same. The modifications in question however offer no special difficulty and we may in all cases make the general statement that any Abelian integal can be reduced to the sum of a rational function of $(z, u)$, of an integral of the first kind, of an expression linear in $p$ elementary integrals of the second kind, and of an integral of the third kind, where in our choice of the $p$ elementary integrals of the second kind we are subject to this one limitation only, that an adjoint curve of 
degree $n-3$ shall not exist which-over and above the zeros already implied in the fact of adjointness-possesses zeros corresponding to the several points $\left(a_{\lambda}, b_{\lambda}\right)$ (and more precisely in the case of double points, corresponding to the individual branches), for which our $p$ elementary integrals have been respectively constructed.

To cover all cases we should have to modify the notation employed in the formula (70) to designate an elementary integral of the second kind, as the notation $\operatorname{II}\left(a_{\lambda}, b_{\lambda}\right)$ in the case of a double point does not distinguish between the elementary integrals corresponding to the separate branches, either or both of which may happen to be included in our system of $p$ elementary integrals of the second kind.

Chicago, November 27, 1900. 